$\begin{array}{rrr}\text { SUSX-TH/01-040 } & \text { OUTP-01-57P } & \text { IHES/P/01/53 } \\ \text { LPT-Orsay-01/114 } \quad \text { HWM-01-40 } & \text { EMPG-01-19 } \\ \text { hep-th/0112104 } & \text { December 2001 }\end{array}$

\title{
Conformal Orbifold Partition Functions from Topologically Massive Gauge Theory
}

\author{
P. Castelo Ferreira \\ Center for Theoretical Physics - University of Sussex, Falmer, Brighton BN1 9QJ, U.K. and \\ Dep. de Matemática - Instituto Superior Técnico, Av. Rovisco Pais, 1049-001 Lisboa, Portugal \\ pcf20@pact.cpes.susx.ac.uk \\ I.I. Kogan \\ Dept. of Physics, Theoretical Physics - University of Oxford, Oxford OX1 3NP, U.K., \\ IHES, 35 Route de Chartres, 91440 Bures-sur-Yvette, France, and \\ Lab. de Physique Théorique, Université de Paris XI, 91405 Orsay Cédex, France \\ kogan@thphys.ox.ac.uk \\ R.J. Szabo \\ Dept. of Mathematics - Heriot-Watt University, Riccarton, Edinburgh EH14 4AS, U.K. \\ R.J.Szabo@ma.hw.ac.uk
}

\begin{abstract}
We continue the development of the topological membrane approach to open and unoriented string theories. We study orbifolds of topologically massive gauge theory defined on the geometry $[0,1] \times \Sigma$, where $\Sigma$ is a generic compact Riemann surface. The orbifold operations are constructed by gauging the discrete symmetries of the bulk three-dimensional field theory. Multi-loop bosonic string vacuum amplitudes are thereby computed as bulk correlation functions of the gauge theory. It is shown that the three-dimensional correlators naturally reproduce twisted and untwisted sectors in the case of closed worldsheet orbifolds, and Neumann and Dirichlet boundary conditions in the case of open ones. The bulk wavefunctions are used to explicitly construct the characters of the underlying extended Kac-Moody group for arbitrary genus. The correlators for both the original theory and its orbifolds give the expected modular invariant statistical sums over the characters.
\end{abstract}

PACS: 11.10.Kk, 11.15.Tk, 11.25.Hf, 11.25.Sq

Keywords: Chern-Simons theories, Strings, Conformal field theory, Orbifolds, Statistical sums 


\section{Contents}

\begin{tabular}{llr}
\hline 1 & Introduction & 1
\end{tabular}

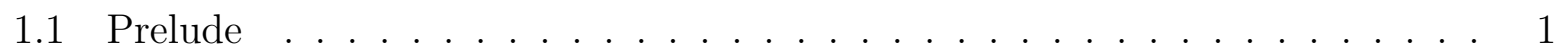

1.2 Summary of Results . . . . . . . . . . . . . . . . . . . 3

2 Canonical Formalism for Topologically Massive Gauge Theories 4

2.1 Hamiltonian Quantization . . . . . . . . . . . . . . . . . . . . 5

2.2 Boundary Partition Functions . . . . . . . . . . . . . . . . . . . . . . . . 7

\begin{tabular}{lll}
\hline 3 & Orbifold Constructions & 10
\end{tabular}

3.1 Field Transformations . . . . . . . . . . . . . . . . . . . . . . . . . . 11

3.2 Orbifold Partition Functions . . . . . . . . . . . . . . . . . . . . . . . . . . . 12

4 Chern-Simons Wavefunctions and Amplitudes $\quad 14$

4.1 Schrödinger Wavefunctionals . . . . . . . . . . . . . . . . . . . . . . . . . . . 14

4.2 Inner Products $\ldots \ldots \ldots \ldots \ldots$. . . . . . . . . . . . . . . 17

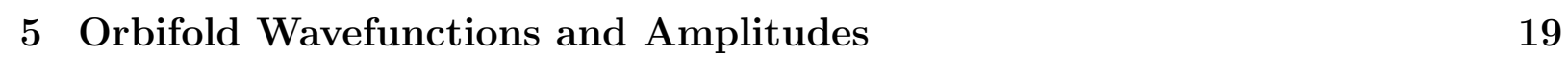

5.1 Orbifold Path Integral . . . . . . . . . . . . . . . . . . . . . . . 20

5.2 Boundary Conditions . . . . . . . . . . . . . . . . . . . . 24

5.3 Normalizations . . . . . . . . . . . . . . . . . . . 26

\begin{tabular}{llr}
6 & Character Expansions & 28 \\
\hline
\end{tabular}

6.1 Effective Topological Wavefunctions . . . . . . . . . . . . . . . . . . . . . . . 28

6.2 Statistical Sums . . . . . . . . . . . . . . . . . . . . . . . . 32

6.3 Examples: One-Loop Amplitudes . . . . . . . . . . . . . . . . . . . . . . . . 35

6.4 Remarks on Modular Invariance . . . . . . . . . . . . . . . . . . . . . . . . 38 


\section{Introduction}

This paper is a continuation of the ongoing development of the topological membrane approach to open and unoriented string theories, in which the focus will be on obtaining the appropriate spectrum and amplitudes of worldsheet orbifold models directly from a threedimensional topologically massive gauge theory. As we will see, this formalism provides a systematic and powerful way of constructing correlation functions in two-dimensional conformal field theories. At the same time, we will obtain a very simple description of conformally invariant twist sectors and boundary conditions. We thereby formulate the intrinsic properties of open and unoriented string theories within the much simpler setting of three-dimensional quantum gauge theory.

\section{$1.1 \quad$ Prelude}

Although open strings were originally considered as defining theories by themselves, it soon become evident that they must come accompanied by closed (non-chiral) strings, in order to maintain unitarity of the string theory. Furthermore, open string theories may be obtained from closed ones by gauging certain discrete geometrical symmetries of the closed string theory [1] [10. The simplest instance uses worldsheet parity $\Omega: z \mapsto-\bar{z}$ which imposes the identification $\sigma_{1} \equiv-\sigma_{1}$ of the worldsheet coordinates, where $z=\sigma_{1}+i \sigma_{2}$ and $\bar{z}=\sigma_{1}-i \sigma_{2}$ define the complex structure of the worldsheet. The spaces obtained in this way can be either closed and unoriented, or open and oriented (and unoriented as well). The latter worldsheets are generally called orbifolds and the singular points of the orbifold projection become the boundaries of the open string worldsheet. The physical states (or more generally the quantum fields) of the open or unoriented theory are obtained from those of the closed oriented theory by projecting out the ones which have negative worldsheet parity eigenvalues. This is accomplished by building a suitable projection operator that retains only the states of even parity in the theory.

Another construction in string theory involves orbifolding the target space of the theory with respect to an involution of some spacetime symmetry. In this paper we will only consider involutions which are of order 2 , obtained by imposing the identifications $X^{I} \equiv$ $-X^{I}$ on the target space coordinates. Upon combining both worldsheet and target space orbifold constructions, i.e. $X^{I}(z, \bar{z}) \equiv-X^{I}(-\bar{z},-z)$, we obtain open or unoriented theories in orbifolds 8 10]. In other words, one arrives at orientifolds, leading to twisted sectors in the Hilbert space of the open or unoriented theories. An important result implied by these constructions is that the gauge group of the open string theory, describing the Chan-Paton degrees of freedom carried by the target space photon Wilson lines, is constrained [11 15. This constraint is closely related to the types of boundary conditions that one must impose 
on insertions of boundary vertex operators at the level of the worldsheet field theory [16].

The perturbation expansion of a string theory is a sum over the genus $g$ of the worldsheet $\Sigma$. Each term in the series yields string scattering amplitudes for a particular order $g$. The object one needs to compute to deal with the genus expansion is the partition function, or in other words the vacuum-to-vacuum amplitudes order by order, i.e. for each genus $g$. In this paper we will compute these objects using the topological membrane approach to string theory [17 35], which consists of replacing the string worldsheet by a three-dimensional manifold. Dynamically, it is modelled by a topologically massive gauge theory [36 39], i.e. the gauge theory whose action contains both a Yang-Mills and a Chern-Simons term for the gauge fields living on a three-manifold, together with topologically massive gravity, i.e. the three-dimensional gravitational theory whose action contains both an Einstein and a gravitational Chern-Simons term. The membrane is the three-dimensional manifold $M=[0,1] \times \Sigma$ which has two disjoint boundaries $\partial M=\Sigma_{0} \amalg \Sigma_{1}$. The gauge non-invariance of the resulting theory induces chiral conformal field theories on the boundaries. The advantage of this approach is that many stringy properties have very transparent and simple physical characterizations in terms of three-dimensional gauge and gravity theories. The original work on this subject concerned only pure Chern-Simons theories in the bulk [38 45].

In this setting, closed string theories are obtained as the effective boundary theories with worldsheet the closed surface $\partial M$. Obtaining an open string theory raises a problem, because in that case one needs an open worldsheet. For a smooth manifold the boundary of a boundary is empty, $\partial \partial M=\emptyset$, and so naively it seems that topological membrane theory cannot describe open strings since the induced worldsheets are already the boundary of a three-manifold. The solution to this problem is to consider an orbifold of the bulk theory. The fixed points of the orbifold then play the role of the one-dimensional boundary of the two-dimensional boundary of the three-dimensional membrane. This approach was first suggested in 45] within the context of pure Chern-Simons gauge theories, and developed further in [33, 34]. There the discrete symmetries of the three-dimensional theory, namely $\Omega T$ and $\Omega C T$, are used as the generators of the orbifold group.

Various other works have also gone in this direction. Recent studies on Wess-ZuminoNovikov-Witten (WZNW) orbifold constructions can be found in 46 48. In 49, D-branes are described at the worldsheet level as vertex operators. Studies of generic rational conformal field theories with boundaries from pure three-dimensional Chern-Simons theory appear in 50 52]. Recent developments related to the topics covered in this paper may also be found in 53 57. 


\subsection{Summary of Results}

This paper continues and develops further the study started in [33, 34]. The main objective is to compute the partition functions of two-dimensional open and unoriented conformal field theories from the perspective of a three-dimensional topological membrane. For simplicity, we will consider here only a $U(1)$ topologically massive gauge theory whereby everything can be worked out explicitly. This corresponds to the simplest instance of bosonic strings compactified on a circle, although the techniques introduced and conclusions reached also apply to more complicated situations. In particular, we will reproduce the standard modular invariants corresponding to a $U(1)$ affine Lie algebra. Some of these results were announced in [34] where a general review may also be found.

In the following we will interpret the boundary partition function as the bulk vacuumto-vacuum amplitude. This translates into computing boundary-to-boundary correlators $\left\langle\Psi_{0} \mid \Psi_{1}\right\rangle$ of ground state wavefunctions [41, 42]. The wavefunctions of topologically massive gauge theory [58, inserted at each of the boundaries, work as chiral WZNW models [44] and render the theory well-defined by effectively enforcing the appropriate boundary conditions on the full three-dimensional gauge theory [34, 38]. One also has to consider the monopole effects described in [32] in order to get the correct holomorphic-antiholomorphic pairing of the chiral conformal blocks. However, these boundary insertions must be suitably modified, because we want to orbifold the bulk theory, but the wavefunctions only live at the boundaries. The solution is to extend them appropriately to the full three-dimensional spacetime. Once this is accomplished we can generate the orbifold with a new boundary at the orbifold plane, and thereby obtain a new wavefunction which we show has to be the constant state $|1\rangle$ such that the correlation function becomes $\left\langle\Psi_{0} \mid 1\right\rangle$.

In the case that the orbifold boundary is an open surface, the new induced wavefunction is not quite unity. There are also induced factors resulting from the functional integration on the new surface which are responsible for setting the correct conformal boundary conditions for each orbifold, namely Dirichlet for $\Omega T$ and Neumann for $\Omega C T$. It is necessary in this description to consider a one-dimensional boundary correction to the Gauss law upon orbifolding. It is also through this term that insertions of vertex operators in the induced conformal field theory [49, 56, 57] can be described by the topological membrane [59], particularly for Dirichlet boundary conditions since in that case the source terms at the orbifold fixed points couple to the gauge field and act as vertex operators on the boundary. In fact, this constitutes the first step towards the construction of D-branes from topological membrane theory [59]. In this way we will explicitly compute, for any genus $g$, the string vacuum amplitudes entirely from the topological membrane. They are expressed as modular invariant combinations of affine characters of the two-dimensional conformal field theory, which we compute from the three-dimensional gauge theory by appropriately incor- 
porating the remaining homology dependence of the wavefunctions on the Riemann surface $\Sigma$. An unsolved problem that remains concerns the construction of more general modular invariants. Within the present framework this is is not possible without a mechanism which creates a tunnelling effect between various ground state wavefunctions that is more general than the one described in [32. For this, it is most likely necessary to take into account the effects of gravity in the bulk theory [22, 35, 60].

The organization of the remainder of this paper is as follows. In the next section we review the essential features of topologically massive gauge theories that we shall need and outline how they will be used to construct the modular invariant partition functions of conformal field theories. In section 3 we summarize the three-dimensional orbifold operations that will be used and how they will naturally induce the amplitudes appropriate to the orbifold conformal field theories. In section 1 we explicitly construct the vacuum wavefunctions of topologically massive gauge theory and use them to study the bulk partition function. In section 5 we then consider the effects of orbifolding on these wavefunctions and amplitudes. Finally, in section 6 we carefully examine the topological dependence of the gauge theory wavefunctions and derive very explicit expressions for the conformal field theory modular invariants valid for arbitrary genus $g$. The case of genus one is studied in some detail for illustration.

\section{Canonical Formalism for Topologically Massive Gauge Theories}

In this section we will review some of the basic aspects of $U(1)$ topologically massive gauge theories [36 39] that will be required in the following. The gauge theory is defined on a three-dimensional manifold of the form $M=[0,1] \times \Sigma$, where the finite interval $[0,1]$ parametrizes the time $t$, and $\Sigma$ is a compact Riemann surface whose local complex coordinates will be denoted $\mathbf{z}=(z, \bar{z})$ with integration measure $d^{2} z=|d z \wedge d \bar{z}|$. We will adopt Gaussian normal coordinates for the Minkowski three-geometry, in which the metric takes the form

$$
d s_{(3)}^{2}=g_{\mu \nu} d x^{\mu} d x^{\nu}=-d t^{2}+h_{i j} d x^{i} d x^{j} .
$$

The three-manifold has two boundaries $\Sigma_{0}$ and $\Sigma_{1}$ at times $t=0$ and $t=1$, respectively, both of which are copies of $\Sigma$ with opposite orientation. The action is a sum of Maxwell and Chern-Simons terms for a $U(1)$ gauge field $A$ with curvature $F$,

$$
S_{\mathrm{TMGT}}[A]=\int_{0}^{1} d t \int_{\Sigma} d^{2} z\left[-\frac{\sqrt{-g}}{4 \gamma} F_{\mu \nu} F^{\mu \nu}+\frac{k}{8 \pi} \epsilon^{\mu \nu \lambda} A_{\mu} \partial_{\nu} A_{\lambda}+\sqrt{-g} A_{\mu} J^{\mu}\right]
$$


where we have included the minimal coupling of the gauge fields to a conserved current $J^{\mu}$,

$$
\partial_{\mu} J^{\mu}=0
$$

The bulk Levi-Civita antisymmetric tensor density $\epsilon^{\mu \nu \lambda}$ induces the tensor density $\epsilon^{i j}=\epsilon^{0 i j}$ on the boundaries. As has been extensively studied in the past [17 21, 23, 35, 40 43], the quantum field theory defined by (2.2) induces new degrees of freedom on the boundaries which constitute chiral WZNW models. They are fields belonging to two-dimensional chiral conformal field theories living on $\Sigma_{0}$ and $\Sigma_{1}$.

\subsection{Hamiltonian Quantization}

The action (2.2) can be written in a canonical splitting $A_{\mu}=\left(A_{0}, A_{i}\right)$ as

$$
\begin{aligned}
S_{\mathrm{TMGT}}[A]= & \int_{0}^{1} d t \int_{\Sigma} d^{2} z\left[-\frac{\sqrt{-g}}{2 \gamma} F_{0 i} F^{0 i}-\frac{\sqrt{-g}}{4 \gamma} F_{i j} F^{i j}+\frac{k}{16 \pi} \epsilon^{i j} A_{0} F_{i j}+\frac{k}{8 \pi} \epsilon^{i j} A_{i} F_{j 0}\right. \\
& \left.+\sqrt{-g} A_{0} J^{0}+\sqrt{-g} A_{i} J^{i}\right] .
\end{aligned}
$$

The canonical momentum conjugate to $A_{i}$ is

$$
\Pi^{i}=-\frac{\sqrt{-g}}{\gamma} F^{0 i}+\frac{k}{8 \pi} \epsilon^{i j} A_{j},
$$

while, as usual, the canonical momentum conjugate to $A_{0}$ is identically zero. The field $A_{0}$ is therefore non-dynamical and serves as a Lagrange multiplier which imposes the Gauss law constraint

$$
0=\int_{\Sigma} d^{2} z\left(-\frac{\sqrt{h}}{\gamma} \partial_{i} F^{0 i}+\frac{k}{8 \pi} \epsilon^{i j} F_{i j}+\sqrt{h} J^{0}\right)-\oint_{\partial \Sigma}\left(-\frac{\sqrt{h}}{\gamma} F^{0 i}+\frac{k}{8 \pi} \epsilon^{i j} A_{j}\right) n_{i}
$$

where $n_{i}$ is a vector normal to the boundary of $\Sigma$. Note that the boundary term in (2.6) is only present when the two-dimensional boundary $\Sigma$ of the underlying three-manifold has a boundary. Of course for a smooth space this term doesn't appear because the boundary of a boundary is empty. However, once we quotient the theory by its discrete symmetries new boundaries can emerge at orbifold singularities [33, 34, 45]. This extra boundary term is vital for the construction we will present in the following, because it allows the imposition of the correct boundary conditions on the conformal field theory. Moreover, conformal vertex operators inserted on the boundary are thereby included in the full threedimensional theory as external fluxes coupled to the gauge fields through the conserved current $J^{\mu}$ [59], in accordance with the fact that closed string vertex operators correspond to Wilson lines of the three-dimensional gauge theory. We will see later on that the external 
charges actually allow one to introduce collective coordinates of D-branes [59]. It is also this mechanism that constrains the open string gauge group and therefore the Chan-Paton degrees of freedom [12,14].

The Hamiltonian of the theory is given by

$$
\begin{aligned}
H= & \int_{\Sigma} d^{2} z\left\{-A_{0}\left[\partial_{i}\left(\Pi^{i}-\frac{k}{8 \pi} \epsilon^{i j} A_{j}\right)+\frac{k}{4 \pi} \epsilon^{i j} \partial_{i} A_{j}+\sqrt{h} J^{0}\right]\right. \\
& +\partial_{i}\left[A_{0}\left(\Pi^{i}+\frac{k}{8 \pi} \epsilon^{i j} A_{j}\right)\right]+\frac{1}{8 \gamma \sqrt{h}}\left(\epsilon^{i j} F_{i j}\right)^{2} \\
& \left.+\frac{\gamma}{2 \sqrt{h}} h_{i j}\left(\Pi^{i}-\frac{k}{8 \pi} \epsilon^{i k} A_{k}\right)\left(\Pi^{j}-\frac{k}{8 \pi} \epsilon^{j l} A_{l}\right)-\sqrt{h} A_{i} J^{i}\right\} .
\end{aligned}
$$

Defining the electric and magnetic fields as

$$
\begin{aligned}
E^{i} & =-\frac{1}{\gamma} F^{0 i} \\
B & =\partial_{z} A_{\bar{z}}-\partial_{\bar{z}} A_{z}
\end{aligned}
$$

the Gauss law constraint (without boundary terms) reads

$$
\partial_{i} E^{i}+\frac{k}{4 \pi} B=-J^{0}
$$

In the quantum field theory, the canonical commutation relations can be written as

$$
\begin{aligned}
{\left[E^{i}(\mathbf{z}), E^{j}\left(\mathbf{z}^{\prime}\right)\right] } & =-i \frac{k}{4 \pi} \epsilon^{i j} \delta^{(2)}\left(\mathbf{z}-\mathbf{z}^{\prime}\right) \\
{\left[E^{i}(\mathbf{z}), B\left(\mathbf{z}^{\prime}\right)\right] } & =-i \epsilon^{i j} \partial_{j} \delta^{(2)}\left(\mathbf{z}-\mathbf{z}^{\prime}\right)
\end{aligned}
$$

and the constraint (2.9) leads to an equation that needs to be satisfied by the physical (gauge invariant) states. We will use this equation in section 0 when we construct the quantum wavefunctions of the field theory. Note that the $A_{0}$ dependent terms in (2.7) vanish when $H$ acts on such states.

The generators of time-independent local gauge transformations can be easily defined, for smooth real-valued gauge parameter functions $\Lambda$, as [25]

$$
U_{\Lambda}=\exp \left\{i \int_{\Sigma} d^{2} z \sqrt{h} \Lambda(\mathbf{z})\left(\partial_{i} E^{i}+\frac{k}{4 \pi} B+J^{0}\right)\right\} .
$$

The physical Hilbert space consists of those quantum states which are invariant under the actions of the operators (2.11). In addition, when there are topologically non-trivial gauge field configurations, we must take into account the large gauge transformations of the theory. These are generated by the operators (2.11) obtained by taking $\Lambda$ to be the 
multi-valued angle function of the Riemann surface $\Sigma$. Then integrating by parts in (2.11) yields the extra local operator 61 64]

$$
V\left(\mathbf{z}_{0}\right)=\exp \left\{-i \int_{\Sigma} d^{2} z\left[\left(E^{i}+\frac{k \sqrt{h}}{4 \pi} \epsilon^{i j} A_{j}\right) \epsilon_{i k} \partial^{k} \ln E\left(\mathbf{z}, \mathbf{z}_{0}\right)-\sqrt{h} \theta\left(\mathbf{z}, \mathbf{z}_{0}\right) J^{0}\right]\right\},
$$

where $E\left(\mathbf{z}, \mathbf{z}_{0}\right)$ is the prime form of $\Sigma$ (see section 6.2 ), $\mathbf{z}_{0}$ is a fixed point on $\Sigma$, and

$$
\theta\left(\mathbf{z}, \mathbf{z}_{0}\right)=\operatorname{Im} \ln \frac{E\left(\mathbf{z}, \mathbf{z}_{0}\right)}{E\left(\mathbf{z}, \mathbf{z}^{\prime}\right) E\left(\mathbf{z}^{\prime}, \mathbf{z}_{0}\right)}
$$

with $\mathbf{z}^{\prime}$ an arbitrary fixed reference point. Demanding invariance under these operators, i.e. under large gauge transformations, further truncates the physical Hilbert space of the quantum field theory. Moreover, from the commutation relations (2.10) we obtain the relation

$$
\left[B(\mathbf{z}), V^{n}\left(\mathbf{z}_{0}\right)\right]=2 \pi n \sqrt{h} V^{n}\left(\mathbf{z}_{0}\right) \delta^{(2)}\left(\mathbf{z}-\mathbf{z}_{0}\right)
$$

for any integer $n$. This means that the operator $V^{n}$ creates a pointlike magnetic vortex at $\mathbf{z}_{0}$ with magnetic flux $\int_{\Sigma} d^{2} z \sqrt{h} B=2 \pi n$. These objects thereby generate nonperturbative processes which constitute monopoles of the gauge theory. Moreover, from Gauss' law (2.9) we see that they also carry a bulk electric charge

$$
\Delta Q=-\frac{n k}{2}
$$

The electric charge spectrum of the quantum field theory is [25]

$$
Q_{m, n}=m+\frac{k}{4} n
$$

where $m$ and $n$ are integers representing, respectively, the contributions from the usual Dirac charge quantization and the monopole flux. Due to the existence of monopole induced processes and linkings between Wilson lines (charge trajectories) it can be shown [32] that, with the correct relative boundary conditions, the insertion of the charge $Q_{m, n}$ at one boundary $\Sigma_{0}$ (corresponding to a vertex operator insertion in the boundary conformal field theory) necessitates an insertion of the charge

$$
\bar{Q}_{m, n}=m-\frac{k}{4} n
$$

at the other boundary $\Sigma_{1}$. This fact will be assumed throughout the rest of this paper.

\subsection{Boundary Partition Functions}

In the following we will explain how to explicitly build orbifolded boundary partition functions of conformal field theory from correlation functions in the three-dimensional gauge 
theory. For this, we will rederive the well-known one-to-one correspondence between the wavefunctions of the gauge theory and the conformal blocks of the boundary conformal field theory [40, 42]. In particular, for rational values

$$
k=\frac{2 p}{q}
$$

of the Chern-Simons coefficient, with $p$ even, there are $(p q)^{g}$ independent wavefunctions corresponding to the conformal blocks of the induced $c=1$ rational conformal field theory, i.e. of the WZNW model that emerges on the boundary $\Sigma$, where $g$ is the genus of $\Sigma$. The gauge theory partition function then takes the form

$$
Z_{\mathrm{TMGT}}=\sum_{\lambda \in\left(\mathbb{Z}_{p q}\right)^{g}}\left\langle\Psi_{\lambda}(0) \mid \Psi_{\lambda}(1)\right\rangle,
$$

where $\Psi_{\lambda}(0)$ and $\Psi_{\lambda}(1)$ denote the wavefunctions at the boundaries $\Sigma_{0}$ and $\Sigma_{1}$, respectively. It is the correct definition of the object (2.19) and its orbifold version that we are going to deal with in this paper.

As explained in the previous subsection, a vertex operator insertion corresponding to a collection of primary charges

$$
\frac{\lambda^{\ell}}{q}=m^{\ell}+\frac{k n^{\ell}}{4}, \quad \ell=1, \ldots, g
$$

at one boundary will correspond at the other boundary to an insertion with charges

$$
\frac{\bar{\lambda}^{\ell}}{q}=m^{\ell}-\frac{k n^{\ell}}{4}, \quad \ell=1, \ldots, g,
$$

where $m^{\ell}$ and $n^{\ell}$ are winding and monopole numbers acquired by a charge as it moves around the canonical homology cycles of the Riemann surface $\Sigma$. Here and in the following we will exploit the fact that a monopole singularity effectively produces a puncture on $\Sigma$ and hence extra non-contractible loops. For ease of notation, we will, without loss of generality, model the extra homology generators using a set of canonical cycles of the surface. The charge non-conserving processes are then determined by the large gauge transformations which wind around these cycles. In terms of the wavefunctions (or conformal blocks) this raises a problem since we have not yet determined how to introduce the monopole processes into the path integral. They can be thought of as insertions of the local Gauss operator $V$ given in (2.12). The main point is that the physical wavefunctions are the ones which are invariant under the actions of all combinations of the operators $V$ 61 64. G Generally, physical states are constructed from a generic one $|\Psi\rangle$ through a projection onto gauge

\footnotetext{
${ }^{1}$ Thanks to Alex Kovner for this useful remark.
} 
invariant states, which may be realized explicitly by integrating over all gauge copies of the state,

$$
\mathcal{P}|\Psi\rangle=\frac{1}{\operatorname{vol} \mathcal{G}} \int D \Lambda U_{\Lambda}|\Psi\rangle
$$

where $\mathcal{G}$ is the group of gauge transformations $\Sigma \rightarrow U(1)$. This means, in particular, that one must include arbitrary monopole processes in all physical amplitudes. Moreover, as shown in [32, the total probability amplitude for the combinations of wavefunctions (at the boundaries) which are not invariant under these operators vanishes.

In this way the effective path integral will have to take into account this phenomenon and be of the form

$$
\begin{aligned}
Z_{\Psi} & =\sum_{\lambda \in\left(\mathbb{Z}_{p q}\right)^{g}}\left\langle\Psi_{\lambda}(0)\left|\prod_{a} V^{n_{a}}\left(\mathbf{z}_{a}\right) \sum_{\lambda^{\prime} \in\left(\mathbb{Z}_{p q}\right)^{g}}\right| \Psi_{\lambda^{\prime}}(1)\right\rangle \\
& =\sum_{\lambda \in\left(\mathbb{Z}_{p q}\right)^{g}}\left\langle\Psi_{\lambda}(0) \mid \Psi_{\bar{\lambda}}(1)\right\rangle .
\end{aligned}
$$

We will not develop this issue in detail here, as it is enough to consider shifts of the charges at $\Sigma_{1}$ by amounts $\Delta Q^{\ell}=-k n^{\ell} / 2$ or $\Delta Q^{\ell}=0$, depending on the relative boundary conditions, with respect to those at $\Sigma_{0}$. The effective wavefunction at the boundary $\Sigma_{1}$ is then given in terms of a Wilson loop as

$$
\Psi_{\bar{\lambda}}(1)=\exp \left\{i \sum_{\ell=1}^{g} \Delta Q^{\ell} \oint_{\beta^{\ell}} A\right\} \Psi_{\lambda}(0)
$$

with $\lambda^{\ell} / q=m^{\ell}+n^{\ell} k / 4$ and $\bar{\lambda}^{\ell} / q=\lambda^{\ell} / q+\Delta Q^{\ell}$, where the contour integrals go around canonical homology cycles $\beta^{\ell}$ associated with the vortex sources on $\Sigma$. These nonperturbative processes, i.e. braidings of Wilson lines and monopole transitions, will take into account the difference in electric charges between the two boundaries $\Sigma_{0}$ and $\Sigma_{1}$.

The free boundary partition function is then of the schematic form

$$
Z_{\text {free }}=\sum_{\lambda \in\left(\mathbb{Z}_{p q}\right)^{g}}\left\langle\Psi_{\lambda}(0) \mid \Psi_{\lambda}(1)\right\rangle+\sum_{\lambda \in\left(\mathbb{Z}_{p q}\right)^{g}}\left\langle\Psi_{\lambda}(0) \mid \Psi_{\bar{\lambda}}(1)\right\rangle .
$$

This is the simplest modular invariant of the boundary conformal field theory. Depending on the relative boundary conditions of the three-dimensional theory, we can set it to be either only the first sum or only the second sum. For free boundary conditions, both sums are present. Generic modular invariants would arise as bilinear combinations

$$
Z_{\zeta}=\sum_{\lambda, \lambda^{\prime}} \zeta_{\lambda \lambda^{\prime}}\left\langle\Psi_{\lambda}(0) \mid \Psi_{\lambda^{\prime}}(1)\right\rangle
$$

of the wavefunctions with non-negative integral coefficients $\zeta_{\lambda \lambda^{\prime}}$. The allowed combinations are specified by the requirement of a modular invariant partition function. Such a 
classification generally requires the inclusion of the gravitational sector of the topological membrane, i.e. the coupling of topologically massive gauge theory to topologically massive gravity. This analysis would take us beyond the scope of the present paper, and thus we shall focus only on the basic modular invariants (2.23).

\section{Orbifold Constructions}

In [33] the procedure to orbifold the topological membrane by gauging the discrete symmetries of topologically massive gauge theory was introduced. In this setting, the original worldsheet $\Sigma$ is regarded as a double cover of an open or unoriented surface $\Sigma^{O}$, which is obtained formally as the quotient $\Sigma^{O}=\Sigma / \mathbb{Z}_{2}$ by an anti-conformal involution $O$ whose fixed points correspond to the boundary points of $\Sigma^{O}$. Of course if $\Sigma^{O}$ is itself oriented and closed, the cover consists of the two disjoint copies $\Sigma_{0}$ and $\Sigma_{1}$ of $\Sigma$ endowed with opposite orientation. When the worldsheet involution is combined with a time reversal operation, we may then regard the worldsheet $\Sigma^{O}$ from the topological membrane perspective as the con-

necting three-manifold $([0,1] \times \Sigma) / \mathbb{Z}_{2}$. If $\Sigma^{O}$ is closed and orientable, then the connecting three-manifold is a cylinder over $\Sigma^{O}$.

Let us recall the discrete symmetries of the theory defined on the geometry $[0,1] \times \Sigma$. They are time reversal $T: t \mapsto 1-t, \mathbf{A} \mapsto-\mathbf{A}$, charge conjugation $C: A_{\mu} \mapsto-A_{\mu}$, and worldsheet parity $\Omega: z \mapsto-\bar{z}$. It is also possible to introduce other definitions of parity, which would lead to different types of orbifold constructions. Due to the presence of both the Maxwell and Chern-Simons terms in the action (2.2), the only possible combinations of these three symmetries which are compatible with the three-dimensional action are $\Omega C T$ and $\Omega T$. Note that only the worldsheet $\Sigma$ feels the parity operation. For example, consider the case of a torus $\Sigma=T^{2}$ of modulus $\tau=\tau_{1}+i \tau_{2}$. Orbifolding it with $\tau=i \tau_{2}$ under $\Omega$ produces an annulus (or a cylinder), while for $\tau=1 / 2+i \tau_{2}$ a Möbius strip is obtained. On the other hand, starting with $\tau=2 i \tau_{2}$ and the orbifold under $\Omega^{\prime}: z \mapsto-\bar{z}+2 \pi i \tau_{2}$, a Klein bottle is produced. See [33] for further details. We will return to these examples in section 6 .

We will use the following notation in the remainder of this paper. We assume a decomposition of the fields into components parallel $(\|)$ and perpendicular $(\perp)$ to the orbifold line. Such a decomposition is introduced because the orbifold line will constitute the new boundary for the open orbifolds such that the fields straightforwardly decompose into tangential and normal components with respect to the boundary. The results can thereby be applied to more general orbifolds. For the torus and its orbifolds the parallel components correspond to the $y$ direction in the plane and the perpendicular ones to the $x$ direction, such that a generic vector $\mathbf{X}$ has complex components $X_{z}=X_{\perp}+i X_{\|}$and $X_{\bar{z}}=X_{\perp}-i X_{\|}$. 


\subsection{Field Transformations}

Under the $\Omega C T$ symmetry the fields transform as

$$
\begin{aligned}
\Omega C T: \quad t & \longmapsto 1-t \\
x_{\perp} & \longmapsto-x_{\perp} \\
\Lambda & \longmapsto-A_{0} \\
A_{0} & \longmapsto-A_{\perp} \\
A_{\perp} & \longmapsto A_{\|} \\
A_{\|} & \longmapsto-\partial_{i} E^{i} \\
\partial_{i} E^{i} & \longmapsto-B \\
B & \longmapsto Q_{m, n} \\
Q_{m, n} & \longmapsto
\end{aligned}
$$

The orbifold obtained from the quotient under this symmetry corresponds to Neumann boundary conditions on the string fields which restricts the charge spectrum to only KaluzaKlein modes $Q_{m, 0}=m$. For $\Sigma=T^{2}$ and $\tau=i \tau_{2}$ this induces a two-dimensional open conformal field theory living on an annulus $C^{2}$ at the boundaries $\Sigma_{0}$ and $\Sigma_{1}$.

For the $\Omega T$ symmetry the fields transform as

$$
\begin{array}{rll}
\Omega T: \quad t & \longmapsto & 1-t \\
x_{\perp} & \longmapsto & -x_{\perp} \\
\Lambda & \longmapsto & -\Lambda \\
A_{0} & \longmapsto & A_{0} \\
A_{\perp} & \longmapsto & A_{\perp} \\
A_{\|} & \longmapsto & -A_{\|} \\
\partial_{i} E^{i} & \longmapsto & \partial_{i} E^{i} \\
B & \longmapsto & B \\
Q_{m, n} & \longmapsto-Q_{m, n}
\end{array}
$$

The orbifold from the quotient under this symmetry corresponds to Dirichlet boundary conditions on the string fields which restricts the charge spectrum to only winding modes $Q_{0, n}=k n / 4$. Again for $\Sigma=T^{2}$ and $\tau=i \tau_{2}$ this induces a two-dimensional open conformal field theory living on an annulus $C^{2}$ at the boundaries $\Sigma_{0}$ and $\Sigma_{1}$.

Regarding the orbifold operations from the perspective of the full three-dimensional gauge theory, we see that a single new boundary is created at the $T$ orbifold fixed point $t=$ $1 / 2$ as depicted in fig. 1. The new boundary $\Sigma_{\frac{1}{2}}$ may now have a boundary corresponding to the orbifold fixed points $x_{\perp}=0$. Furthermore, the presence of $\Sigma_{\frac{1}{2}}$ constrains the remaining gauge theory. This means that the boundary conditions imposed on the fields at the fixed time slice $\Sigma_{\frac{1}{2}}$ will effectively propagate through the bulk and constrain the conformal field theory living on $\Sigma_{0} \equiv \Sigma_{1}$ as well. More specifically, we are working with the boundary 
conditions of the open conformal field theories in the twisted sectors of the corresponding Hilbert space.
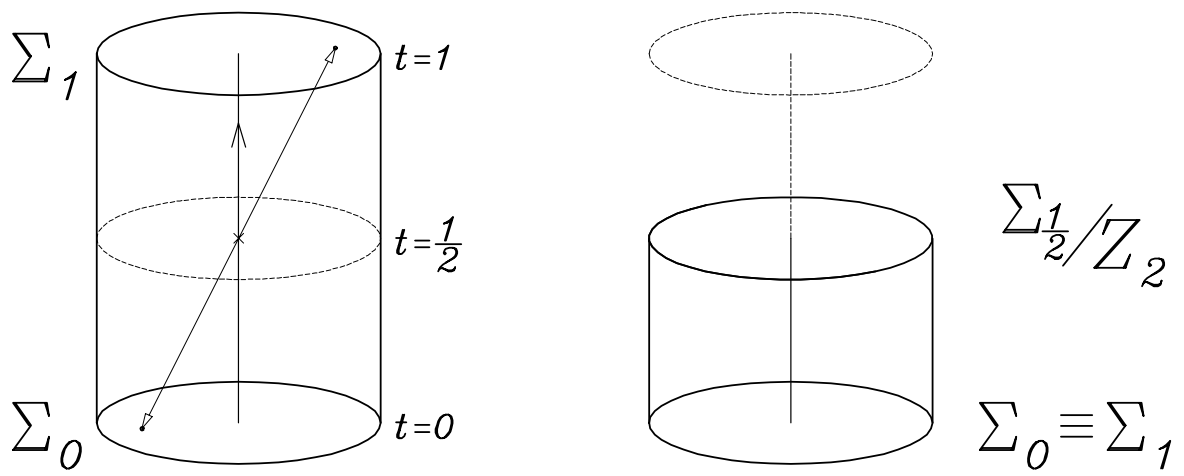

Figure 1: Orbifold of topologically massive gauge theory. The worldsheet $\Sigma_{\frac{1}{2}}$ only feels the discrete symmetries $\Omega C$ and $\Omega$ which each generate the cyclic group $\mathbb{Z}_{2}$.

For later use, we summarize the orbifold identifications on the fixed point surface $\Sigma_{\frac{1}{2}}$ and its boundary $\partial \Sigma_{\frac{1}{2}}$ :

$$
\begin{array}{cccc}
\multicolumn{2}{c}{\Omega \mathbf{T}} & \multicolumn{2}{c}{\Omega \mathbf{C T}} \\
\stackrel{\Sigma_{\frac{1}{2}}}{A_{z} \equiv A_{\bar{z}}} & \frac{\partial \Sigma_{\frac{1}{2}}}{A_{z} \in \mathbb{R}} & A_{z} \bar{\equiv}-A_{\bar{z}} & \frac{\partial \Sigma_{\frac{1}{2}}}{A_{z} \in i \mathbb{R}} \\
A_{\perp} \equiv A_{\perp} & A_{\perp} \neq 0 & A_{\perp} \equiv-A_{\perp} & A_{\perp}=0 \\
A_{\|} \equiv-A_{\|} & A_{\|}=0 & A_{\|} \equiv A_{\|} & A_{\|} \neq 0 .
\end{array}
$$

\subsection{Orbifold Partition Functions}

To deal with the new boundary $\Sigma_{\frac{1}{2}}$ in terms of wavefunctions and partition functions, we insert into (2.23) a complete set of states at time $t=1 / 2$ in order to check compatibility with the orbifolding,

$$
Z_{\Psi}=\sum_{\lambda \in\left(\mathbb{Z}_{p q}\right)^{g}} \sum_{\lambda^{\prime} \in\left(\mathbb{Z}_{p q}\right)^{g}}\left\langle\Psi_{\lambda}(0) \mid \Psi_{\lambda^{\prime}}\left(\frac{1}{2}\right)\right\rangle\left\langle\Psi_{\lambda^{\prime}}\left(\frac{1}{2}\right) \mid \Psi_{\bar{\lambda}}(1)\right\rangle .
$$

We now need to determine which wavefunctions can exist at the orbifold fixed point $t=$ $1 / 2$. For this, let us consider the Wilson line $W_{Q, \mathcal{C}}$ corresponding to an electric charge $Q$ propagating along some oriented path $\mathcal{C}$ in the underlying three-manifold. It transforms under the $\Omega C T$ and $\Omega T$ symmetries as $W_{Q, \mathcal{C}} \mapsto W_{Q,-\mathcal{C}}$ [27, 33], so that under the orbifold operation the identification

$$
W_{Q, \mathcal{C}} \equiv W_{Q,-\mathcal{C}}
$$


must hold for arbitrary contours $\mathcal{C}$. Let us consider, in particular, a path $\mathcal{C}$ which lies entirely within the Riemann surface $\Sigma_{\frac{1}{2}}$, i.e. at fixed time $t=1 / 2$. In order that such Wilson lines be compatible with the orbifold construction they must have vanishing charge, $Q=0$, or else (3.5) is violated. We now recall that the boundary wavefunctions are generated by Wilson lines that live in the surface $\Sigma$ (see (2.24) and [41, 42]). Since the only such Wilson line which is compatible with the orbifold operation is $W_{Q=0, \mathcal{C}}$, the only wavefunction that can live at $\Sigma_{\frac{1}{2}}$ is $\left|\Psi_{\lambda=0}\left(\frac{1}{2}\right)\right\rangle=|1\rangle$.

The orbifold partition function is therefore given by

$$
\begin{aligned}
Z_{\text {orb }} & =\sum_{\lambda \in\left(\mathbb{Z}_{p q}\right)^{g}}\left\langle\Psi_{\lambda}(0)\left|\pi^{\Omega}\right| \Psi_{\bar{\lambda}}(1)\right\rangle \\
& =\sum_{\lambda \in\left(\mathbb{Z}_{p q}\right)^{g}}\left\langle\Psi_{\lambda}^{\text {orb }} \mid 1\right\rangle
\end{aligned}
$$

where

$$
\pi^{\Omega}=\frac{1}{2}(\mathbb{1}+\Omega)
$$

is the orthogonal projection onto parity-even states, i.e. states which are invariant under the interchange of left and right moving worldsheet modes, and $\Psi_{\lambda}^{\text {orb }}=\Psi_{\lambda}(0) \equiv \Psi_{\bar{\lambda}}(1)$. Thus by inserting a projection operator we transform a correlator between two wavefunctions into the correlator between one wavefunction and unity. This result is depicted schematically in fig. 2. It is well-known that there is a one-to-one correspondence between the Hilbert space of physical states of the three-dimensional topological quantum field theory and the space of characters of the induced two-dimensional conformal field theories. The results obtained here are completely consistent with the expressions for conformal field theory partition functions in terms of sums over characters of the conformal group. For closed oriented worldsheets it is a bilinear form in the characters $\chi_{\lambda}$,

$$
\begin{aligned}
Z_{\Psi} & =\left\langle\Psi_{0} \mid \Psi_{1}\right\rangle \\
& =\sum_{\lambda \in\left(\mathbb{Z}_{p q}\right)^{g}} \overline{\chi_{\bar{\lambda}}} \chi_{\lambda},
\end{aligned}
$$

while for open or unoriented worldsheets it is simply linear in the characters,

$$
\begin{aligned}
Z_{\text {orb }} & =\left\langle\Psi_{0}^{\text {orb }} \mid 1\right\rangle \\
& =\sum_{\lambda \in\left(\mathbb{Z}_{p q}\right)^{g}} \chi_{\lambda} .
\end{aligned}
$$

We will see some explicit examples later on. 


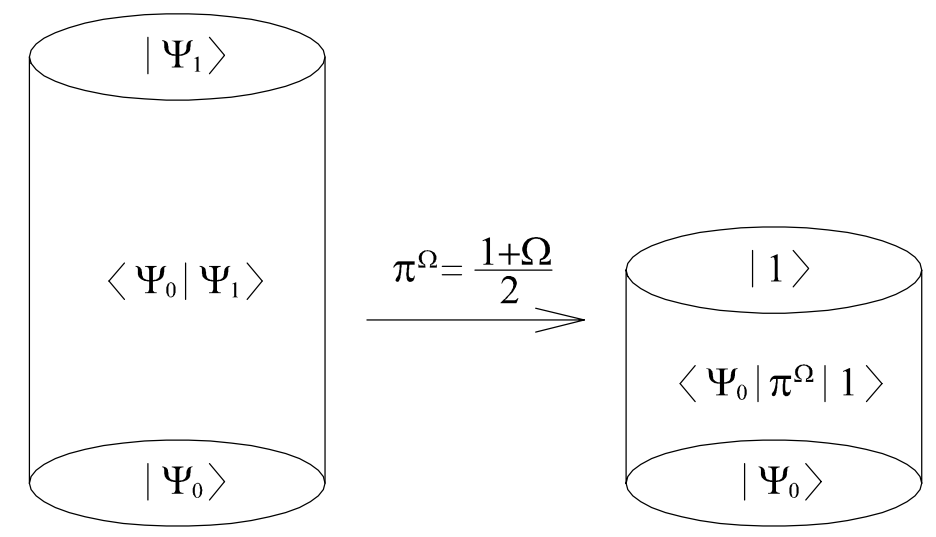

Figure 2: The resulting orbifold construction in terms of wavefunction correlators for the gauged parity symmetry $\Omega$.

\section{Chern-Simons Wavefunctions and Amplitudes}

In this section we will construct the quantum wavefunctions of the three-dimensional gauge theory and use them to study the partition function appropriate to the topological membrane.

\subsection{Schrödinger Wavefunctionals}

Let us examine the topologically massive gauge theory in the functional Schrödinger picture whereby the physical states are the wavefunctionals $\Psi\left[A_{i}\right]$ [58. By using the canonical quantum commutators $(2.10)$ and the representation

$$
\Pi^{i}=-i \sqrt{h} \frac{\delta}{\delta A_{i}},
$$

we impose Gauss' law (2.9) as a constraint equation on the wavefunctionals, which takes the form

$$
\left[\partial_{\bar{z}}\left(-i \frac{\delta}{\delta A_{\bar{z}}}+\frac{k}{8 \pi} \tilde{\epsilon}^{z \bar{z}} A_{z}\right)+\partial_{z}\left(-i \frac{\delta}{\delta A_{z}}-\frac{k}{8 \pi} \tilde{\epsilon}^{z \bar{z}} A_{\bar{z}}\right)+\frac{k}{4 \pi} \tilde{\epsilon}^{z \bar{z}} F_{z \bar{z}}+J^{0}\right] \Psi\left[A_{z}, A_{\bar{z}}\right]=0
$$

where the two-dimensional antisymmetric tensor $\tilde{\epsilon}$ is induced from the bulk by $\tilde{\epsilon}^{i j}=$ $\epsilon^{0 i j} / \sqrt{-g}$. By applying the Hamiltonian (2.7) to such physical wavefunctions in this polarization, we find that the stationary states satisfy the functional Schrödinger equation

$$
\begin{aligned}
\int_{\Sigma} d^{2} z & \sqrt{h}\left\{-\frac{\gamma}{2} h_{z \bar{z}}\left(-i \frac{\delta}{\delta A_{\bar{z}}}-\frac{k}{8 \pi} \tilde{\epsilon}^{z \bar{z}} A_{z}\right)\left(-i \frac{\delta}{\delta A_{z}}+\frac{k}{8 \pi} \tilde{\epsilon}^{z \bar{z}} A_{\bar{z}}\right)\right. \\
+ & \left.\frac{1}{8 \gamma}\left(\tilde{\epsilon}^{z \bar{z}} F_{z \bar{z}}\right)^{2}-A_{z} J^{\bar{z}}-A_{\bar{z}} J^{z}\right\} \Psi\left[A_{z}, A_{\bar{z}}\right]=\mathcal{E} \Psi\left[A_{z}, A_{\bar{z}}\right]
\end{aligned}
$$


where $\mathcal{E}$ is the energy of the state, and we have appropriately normal ordered the Hamiltonian density. Since the gauge constraint commutes with the Hamiltonian, these two equations can be consistently solved.

Because of the appearence of the magnetic field in (2.11), in topologically massive gauge theory the physical states are not gauge invariant. Instead, by integrating the infinitesimal gauge constraint one finds that the gauge symmetry is represented projectively on wavefunctionals as

$$
U_{\Lambda} \Psi\left[A_{i}\right]=\mathrm{e}^{i \alpha\left[A_{i}, \Lambda\right]} \Psi\left[A_{i}+\partial_{i} \Lambda\right]
$$

where the projective phase is given by the one-cocycle

$$
\alpha\left[A_{i}, \Lambda\right]=\frac{k}{8 \pi} \int_{\Sigma} d^{2} z \epsilon^{i j} A_{i} \partial_{j} \Lambda
$$

To separate out the gauge invariant part, one integrates the corresponding cocycle condition such that the physical states decompose into three factors as

$$
\Psi\left[A_{z}, A_{\bar{z}}\right]=\exp \left\{-\frac{i k}{8 \pi} \int_{\Sigma} d^{2} z \sqrt{h} \tilde{\epsilon}^{z \bar{z}} A_{z} A_{\bar{z}}\right\} \psi\left[A_{z}\right] \Phi[B],
$$

where $B$ is the magnetic field.

The factor $\Phi[B]$ is the gauge-invariant solution of Gauss' law for the pure Maxwell theory $(k=0)$,

$$
\left[\partial_{\bar{z}} \frac{\delta}{\delta A_{\bar{z}}}+\partial_{z} \frac{\delta}{\delta A_{z}}\right] \Phi=0
$$

If the fields have non-trivial magnetic charge $\int_{\Sigma} d^{2} z \sqrt{h} B \neq 0$, then the wavefunction $\Phi[B]$ vanishes [58. This result is simply the statement that there is overall charge conservation on the closed surface $\Sigma$. Of course, locally non-zero magnetic field distributions are possible. The component $\psi\left[A_{z}\right]$ obeys the functional Schrödinger equation for the source-coupled, pure Chern-Simons theory $(\gamma \rightarrow \infty)$,

$$
\left[\partial_{z} \frac{\delta}{\delta A_{z}}-\frac{i k}{4 \pi} \tilde{\epsilon}^{z \bar{z}} \partial_{\bar{z}} A_{z}-i J^{0}\right] \psi\left[A_{z}\right]=0 .
$$

In the following we will only be interested in the ground state of the theory. This corresponds to a projection onto the lowest Landau level of the quantum spectrum, which is attained in the topological limit $\gamma \rightarrow \infty$ (the mass gap between Landau levels is proportional to $\gamma$ ). In this case, $\Phi=1$ and we recover the wavefunctions of pure Chern-Simons gauge theory. These solutions correspond to configurations with weak magnetic field, $\tilde{\epsilon}^{z \bar{z}} F_{z \bar{z}} \simeq 0$, and also weak external flux. The stationary states are the eigenfunctions of the first term 
on the left-hand side of (4.3). In particular, the vacuum state $(\mathcal{E}=0)$ is determined by the zero mode equation

$$
\left[\frac{\delta}{\delta A_{z}}-\frac{k}{8 \pi} A_{\bar{z}}\right] \Psi\left[A_{z}, A_{\bar{z}}\right]=0
$$

where we have fixed a complex structure on $\Sigma$ determined by the worldsheet metric whereby $\tilde{\epsilon}^{z \bar{z}}=i$. Furthermore, by using (4.8) and (4.6) with $\Phi=1$, one finds that it obeys the equation

$$
\left[\partial_{\bar{z}} \frac{\delta}{\delta A_{\bar{z}}}+\frac{k}{8 \pi} \partial_{\bar{z}} A_{z}-\frac{k}{4 \pi} F_{\bar{z} z}-J^{0}\right] \Psi\left[A_{z}, A_{\bar{z}}\right]=0
$$

A solution of (4.9) and (4.10), which is compatible with the gauged WZNW construction for an abelian gauge group, is given by a path integral over an auxilliary worldsheet field $\varphi$ as 44

$$
\Psi\left[A_{z}, A_{\bar{z}}\right]=\int D \mu(\varphi) \exp \left\{\int_{\Sigma} d^{2} z\left[\frac{k}{8 \pi}\left(A_{\bar{z}} A_{z}-2 A_{\bar{z}} \partial_{z} \varphi+\partial_{\bar{z}} \varphi \partial_{z} \varphi\right)-J^{0} \varphi\right]\right\}
$$

where the functional integration measure is given by

$$
D \mu(\varphi)=D \varphi \sqrt{\mathcal{A}_{\Sigma}} \delta\left(\int_{\Sigma} d^{2} z \varphi(\mathbf{z}) \phi_{0}\right)
$$

with $\mathcal{A}_{\Sigma}=\int_{\Sigma} d^{2} z \sqrt{h}$ the area of $\Sigma$.

That (4.11) solves (4.10) follows from noting that the integrand of the left-hand side of (4.10) can then be written as a total functional $\varphi$-derivative of the exponential in (4.11), and thereby integrated by parts to zero in $\varphi$ space. The factors in the integration measure (4.12) remove the zero modes of the field $\varphi$ on the Riemann surface $\Sigma$, which is required for a well-defined functional integral because, by charge conservation on a compact space, the exponential in (4.11) is independent of them. Here $\phi_{0}=1 / \sqrt{\mathcal{A}_{\Sigma}}$ is the normalized zero mode eigenfunction of the scalar Laplace operator $\nabla_{\Sigma}^{2}$ on $\Sigma$, and the delta-function in (4.12), whose argument is the coefficient of $\phi_{0}$ in an arbitrary field configuration, projects out the zero mode integration from the measure $D \varphi$. The worldsheet area factor is included to make the overall combination dimensionless. A more convenient way to use this measure is to change variables from $\varphi$ to its worldsheet derivatives and compute the Jacobian to get

$$
D \mu(\varphi)=D\left(\partial_{z} \varphi\right) D\left(\partial_{\bar{z}} \varphi\right) \sqrt{\frac{\mathcal{A}_{\Sigma}}{\operatorname{det}^{\prime} \nabla_{\Sigma}^{2}}}
$$

where the prime on the determinant means that zero-modes are excluded. We will see that the wavefunctions (4.11) are the building blocks of the boundary theories, in that by inserting such states on the boundaries they act as boundary conditions and effectively select the 
boundary world. Through the fields $\varphi$ we are introducing new degrees of freedom on the boundaries which are absolutely necessary for the consistency of the full three-dimensional theory (on a manifold with boundary) as a well-defined gauge theory.

\subsection{Inner Products}

In [41, 44] the partition function is defined through the inner product $\left\langle\Psi_{1} \mid \Psi_{2}\right\rangle$ of wavefunctions at a fixed slice $\Sigma$ of a three-manifold $M$. One chooses a Heegaard splitting $M=M_{1} \#_{\Sigma} M_{2}$ of the manifold such that the boundaries of the two components are identified, up to a homeomorphism, as $\Sigma=\partial M_{1} \cong \partial M_{2}$. However, the present topological membrane case (for which $M=[0,1] \times \Sigma$ ) is somewhat different since the partition function actually represents a quantum correlation between two different states, living at times $t=0$ and $t=1$, corresponding to the two boundaries $\Sigma_{0}$ and $\Sigma_{1}$. We can either interpret it as the amplitude for time evolution of a state inserted at one boundary to the other boundary, or as the correlator of two states defined for every time slice whereby each state decays on different boundaries. This latter interpretation is the approach that we will follow in this paper.

Let us consider two states of the form (4.11) inserted at the boundaries $\Sigma_{0}$ and $\Sigma_{1}$. The partition function for the topological limit of the system is then the correlation function

$$
\begin{aligned}
Z_{\Psi} & =\left\langle\Psi_{0} \mid \Psi_{1}\right\rangle \\
& =\int D \mu_{G}\left(A_{z}, A_{\bar{z}}\right) \mathrm{e}^{i S_{\mathrm{TMGT}}[A]} \overline{\Psi_{0}\left[A_{z}, A_{\bar{z}}\right]} \Psi_{1}\left[A_{z}, A_{\bar{z}}\right],
\end{aligned}
$$

where $D \mu_{G}\left(A_{z}, A_{\bar{z}}\right)$ is the gauge fixed functional integration measure for the gauge fields whose precise form will be given in the next section. The wavefunctional $\Psi_{1}$ is given by (4.11), while $\overline{\Psi_{0}}$ is given by

$$
\overline{\Psi_{0}\left[A_{z}, A_{\bar{z}}\right]}=\int D \mu(\varphi) \exp \left\{-\int_{\Sigma} d^{2} z\left[\frac{k}{8 \pi}\left(A_{\bar{z}} A_{z}-2 A_{z} \partial_{\bar{z}} \varphi+\partial_{\bar{z}} \varphi \partial_{z} \varphi\right)-J^{0} \varphi\right]\right\}
$$

where the overall minus sign comes from the relative change in orientation between the surfaces $\Sigma_{0}$ and $\Sigma_{1}$. As we will now demonstrate, the importance of the insertions of these boundary wavefunctions lies in the fact that they constrain the theory, assuring that the path integral (2.19) is both gauge invariant and has a classical extremum. The quantum field theory would not be consistent without them.

Under a local gauge transformation

$$
\delta_{\Lambda} A_{i}=\partial_{i} \Lambda
$$


the bulk exponential factor in the path integral (4.14) changes according to

$$
\begin{aligned}
\delta_{\Lambda} S_{\mathrm{TMGT}}[A]= & \int_{\Sigma_{1}} d^{2} z\left[\frac{k}{8 \pi}\left(A_{z} \partial_{\bar{z}} \Lambda-A_{\bar{z}} \partial_{z} \Lambda\right)+J^{0} \Lambda\right] \\
& -\int_{\Sigma_{0}} d^{2} z\left[\frac{k}{8 \pi}\left(A_{\bar{z}} \partial_{z} \Lambda-A_{z} \partial_{\bar{z}} \Lambda\right)+J^{0} \Lambda\right] .
\end{aligned}
$$

It is in (4.17) that the gauge non-invariance of the theory resides due to the existence of boundaries, and this leads to chiral WZNW degrees of freedom on the worldsheet $\Sigma$. On the other hand, because of (4.4) the wavefunction (4.11) at the boundary $\Sigma_{1}$ transforms as

$$
\delta_{\Lambda} \Psi_{1}\left[A_{z}, A_{\bar{z}}\right]=\int D \mu(\varphi) \exp \left\{\frac{k}{8 \pi} \int_{\Sigma_{1}} d^{2} z\left[A_{\bar{z}} \partial_{z} \Lambda+A_{z} \partial_{\bar{z}} \Lambda+\partial_{z} \Lambda \partial_{\bar{z}} \Lambda-2 \partial_{z} \varphi \partial_{\bar{z}} \Lambda\right]\right\}
$$

Combining all the factors in (4.17) and (4.18), we find that the $\Lambda$-dependent terms of the gauge variation of (4.14) can be absorbed into the total gauge transformed wavefunction at $\Sigma_{1}$ given by

$$
\begin{aligned}
\Psi_{1}^{\Lambda}\left[A_{z}, A_{\bar{z}}\right]= & \int D \mu(\varphi) \exp \left\{\int _ { \Sigma _ { 1 } } d ^ { 2 } z \left[\frac { k } { 8 \pi } \left(A_{\bar{z}} A_{z}-2 A_{\bar{z}} \partial_{z}(\varphi-\Lambda)\right.\right.\right. \\
& \left.\left.\left.+\partial_{z}(\varphi-\Lambda) \partial_{\bar{z}}(\varphi-\Lambda)\right)-J^{0}(\varphi-\Lambda)\right]\right\} .
\end{aligned}
$$

An analogous result holds for the wavefunction $\overline{\Psi_{0}}$ at the boundary $\Sigma_{0}$. The gauge parameter $\Lambda$ is now easily eliminated by redefining the auxilliary field corresponding to the degree of freedom on the boundary as $\varphi \mapsto \varphi+\Lambda$, such that $\Psi_{1}^{\Lambda}\left[A_{z}, A_{\bar{z}}\right]=\Psi_{1}\left[A_{z}, A_{\bar{z}}\right]$ and $\overline{\Psi_{0}^{\Lambda}\left[A_{z}, A_{\bar{z}}\right]}=\overline{\Psi_{0}\left[A_{z}, A_{\bar{z}}\right]}$. This redefinition does not change the functional integration measure $D \mu(\varphi)$, and thus $\delta_{\Lambda} Z_{\Psi}=1$. It is in this elegant way, by inserting seemingly $a d$ hoc new degrees of freedom, that we manage to ensure gauge invariance of the full theory. In other words, gauge transformations necessarily induce new degrees of freedom on the boundaries, whose dynamics are governed by the chiral $U(1)$ gauged WZNW models (4.11).

Next we show that the boundary wavefunctions ensure that the field theory has a classical extremum. For this, let us consider the case $J^{\mu}=0$ for clarity. An infinitesimal variation of the gauge fields induces a variation of the action of the form

$$
\delta S_{\mathrm{TMGT}}[A, J=0]=\frac{k}{8 \pi}\left(\int_{\Sigma_{1}} d^{2} z \epsilon^{i j} A_{i} \delta A_{j}-\int_{\Sigma_{0}} d^{2} z \epsilon^{i j} A_{i} \delta A_{j}\right) .
$$

From (4.20) it follows that the action $S_{\text {TMGT }}$ has no well-defined classical extremum, because the boundary terms prevent one from setting $\delta S_{\mathrm{TMGT}}=0$. Let us denote the fields $\varphi$ 
corresponding to the worldsheets $\Sigma_{0}$ and $\Sigma_{1}$ by $\varphi_{0}$ and $\varphi_{1}$, respectively. Using the change of variables (4.13), we may integrate the wavefunction product that appears in the partition function (4.14) to get?

$$
\begin{aligned}
Z_{\Psi}[J=0]= & \frac{k \mathcal{A}_{\Sigma}}{\operatorname{det}^{\prime} \nabla_{\Sigma}^{2}} \int D \mu_{G}\left(A_{z}, A_{\bar{z}}\right) \mathrm{e}^{i S_{\mathrm{TMGT}}[A]} \\
& \times \iint D\left(\partial_{\bar{z}} \varphi_{1}\right) D\left(\partial_{z} \varphi_{0}\right) \delta\left(\left.2 A_{\bar{z}}\right|_{\Sigma_{1}}-\partial_{\bar{z}} \varphi_{1}\right) \delta\left(\left.2 A_{z}\right|_{\Sigma_{0}}-\partial_{z} \varphi_{0}\right) \\
& \times \exp \left\{\frac{k}{8 \pi}\left(\int_{\Sigma_{1}} d^{2} z A_{\bar{z}} A_{z}-\int_{\Sigma_{0}} d^{2} z A_{\bar{z}} A_{z}\right)\right\} .
\end{aligned}
$$

In this way the gauge field $A_{\bar{z}}$ is fixed on one boundary while $A_{z}$ is fixed on the other boundary such that $\left.\delta A_{\bar{z}}\right|_{\Sigma_{1}}=\left.\delta A_{z}\right|_{\Sigma_{0}}=0$. The overall boundary variation in (4.21) containing $\delta A_{i}$ vanishes since the term (4.20) which comes from the bulk cancels exactly with the one coming from the boundary exponential term in the wavefunctions. In this way the gauge field theory has a well-defined classical limit. Notice that the fields $\varphi$ living on $\Sigma_{0}$ correspond to holomorphic degrees of freedom while the ones living on $\Sigma_{1}$ correspond to antiholomorphic degrees of freedom. This method of constructing the two chiral conformal field theories on the boundary is thereby equivalent to that described in 23, 32] (see also [34, 44]) by fixing the fields on the boundary and adding extra terms to the boundary action.

\section{Orbifold Wavefunctions and Amplitudes}

In this section we will generalize the analysis of the previous section to the orbifold constructions of section 3 . In order to simplify some arguments which follow we will work with an effective field theory obtained by integrating the bulk dependence of the gauge fields in the path integral. In order to do so we need to gauge fix the functional integral by the standard Faddeev-Popov procedure. For this, we decompose the gauge field $A_{i}$ according to a representative of its gauge orbit as

$$
A_{i}=\bar{A}_{i}+\partial_{i} \Lambda
$$

where $\bar{A}_{i}$ is the gauge-fixed field and $\Lambda$ is an arbitrary real-valued gauge parameter function. Then the measure appearing in (4.14) is defined by

$$
D \mu_{G}\left(A_{z}, A_{\bar{z}}\right)=D \bar{A}_{z} D \bar{A}_{\bar{z}} D \mu(\Lambda) \sqrt{\frac{\operatorname{det}^{\prime} \nabla_{\Sigma}^{2}}{\mathcal{A}_{\Sigma}}} \delta(G(A)) \operatorname{det}\left[\frac{\delta G(A)}{\delta \Lambda}\right],
$$

\footnotetext{
${ }^{2}$ Here and in the following we drop all (infinite) numerical constants via suitable normalizations of the functional integration measures. Other infinite constants are evaluated using standard zeta-function regularization.
} 
where we have used (4.13). Here $G(A)=0$ is the gauge-fixing condition which will be specified later on. As we have seen, the un-orbifolded theory is gauge invariant due to the insertion of new degrees of freedom $\varphi$ on the boundary. Therefore the path integral does not depend on $\Lambda$ and its integration gives an overall (infinite) constant. However, as we will see, this may not be the case once one orbifolds the theory. This is due to the orbifold singularities which play the role of a new boundary of $\Sigma$, and in a very similar way to the process described in 23] a new degree of freedom emerges on the boundary. Put differently, when $\partial \Sigma \neq \emptyset$, one must generalize the analysis of the previous section by solving also the Gauss' law constraint on the boundary of the worldsheet, which appears as the second term in (2.6). As we will show, it will actually be responsible for setting the boundary conditions of the conformal field theory, so that this process picks the correct boundary conditions required by the theory in order to restore gauge invariance.

Assuming that the measure $D \bar{A}_{z} D \bar{A}_{\bar{z}}$ can be factorized into a bulk integration times a boundary integration, we find that the path integral factorizes into a product

$$
Z=Z_{\text {bulk }} Z_{\Psi}
$$

where $Z_{\Psi}$ is the partition function (4.14) containing the boundary wavefunctions. It is evident from the analysis of the previous sections that these two factors are completely decoupled. We may then integrate over the bulk gauge fields and effectively normalize $Z_{\mathrm{bulk}}=1$. Generally, it yields a topological invariant of the underlying framed three-manifold which is constructed from the Chern-Simons invariant, the gravitational $\eta$-invariant, and the RaySinger analytic torsion. The boundary integration over $\bar{A}_{i}$ also factorizes into two contributions, one for each boundary component.

\subsection{Orbifold Path Integral}

Within the framework of the path integral approach to the topological membrane, we can now proceed to orbifold the theory. In order to do so we need information about the wavefunctions for every time $t$, not just for $t=0$ and $t=1$, and in particular at the new boundary at $t=1 / 2$ obtained from the orbifolding. For this, we consider an extension of the exponential integral over $\Sigma$ defining the previous wavefunctions (4.11) and (4.15) to the full three-dimensional manifold $[0,1] \times \Sigma$. The most efficient way to achieve this is to insert two generic time-dependent functions $f_{0}(t)$ and $f_{1}(t)$ with the boundary conditions

$$
\begin{gathered}
f_{0}(0)=-f_{1}(1)=-1, \\
f_{0}(1)=f_{1}(0)=0,
\end{gathered}
$$


and rewrite the wavefunction product appearing in the path integral (4.14) as

$$
\begin{aligned}
\overline{\Psi_{0}\left[A_{z}, A_{\bar{z}}\right]} & \Psi_{1}\left[A_{z}, A_{\bar{z}}\right] \\
= & |\mathcal{N}|^{2} \int D \mu(\varphi) \exp \left\{\int _ { 0 } ^ { 1 } d t \int _ { \Sigma } d ^ { 2 } z \frac { \partial } { \partial t } \left[\left(\frac{k}{8 \pi}\left(A_{\bar{z}} A_{z}+\partial_{\bar{z}} \varphi \partial_{z} \varphi\right)-J^{0} \varphi\right)\left(f_{0}+f_{1}\right)\right.\right. \\
& \left.\left.\quad-\frac{k}{4 \pi}\left(A_{z} \partial_{z} \varphi f_{0}-A_{\bar{z}} \partial_{\bar{z}} \varphi f_{1}\right)\right]\right\}
\end{aligned}
$$

where we have extended the scalar field $\varphi$, previously inserted only at the boundaries through the functional integrals present in the definitions (4.11) and (4.15), to the whole three-manifold $[0,1] \times \Sigma$. We have also introduced a normalization constant $\mathcal{N}$ in the definition of the wavefunctions, which will be fixed later on by certain orthonormality requirements. A possible profile for the temporal functions $f_{0}$ and $f_{1}$ is depicted in fig. 3. Their introduction allows the implementation of the desired program by extending the correlator of two wavefunctions (defined at each time slice) to the full three-dimensional bulk theory.

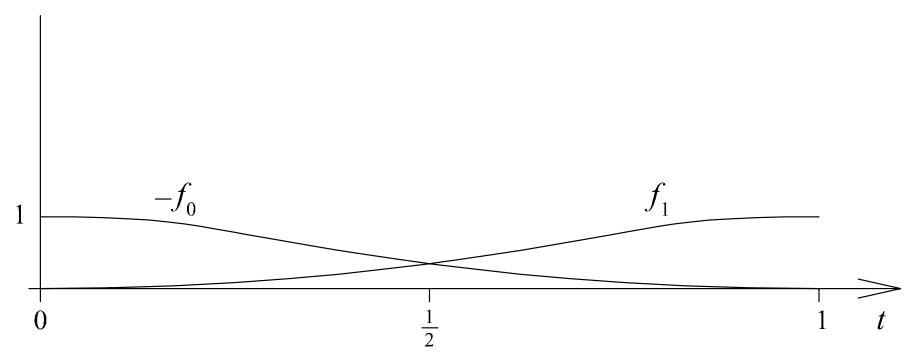

Figure 3: Profile of the decay and growth of the temporal functions $-f_{0}(t)$ and $f_{1}(t)$.

Let us consider the orbifold by the discrete symmetries $\Omega C T$ and $\Omega T$, taking, for consistency, the identifications of the field $\varphi$ to be of the same kind as the gauge parameter $\Lambda$ in section 3.1. Since $t=1 / 2$ is a fixed point of the identification, we split the time integral over $[0,1]$ into two pieces over $\left[0, \frac{1}{2}\right]$ and $\left(\frac{1}{2}, 1\right]$. Rewriting (5.5) with this splitting and performing the required identifications we conclude that, for compatibility with the discrete symmetries, the time-dependent functions must be related by

$$
f_{0}(1-t)=-f_{1}(t)
$$

Putting everything together we find that both parts of the temporal integration give the same contribution. Furthermore, since $f_{0}\left(\frac{1}{2}\right)=-f_{1}\left(\frac{1}{2}\right)$, the product (5.5) reduces under 
the orbifold operation to

$$
\begin{aligned}
\overline{\Psi_{0}^{\text {orb }}\left[A_{z}, A_{\bar{z}}\right]} \Psi_{\frac{1}{2}}^{\text {orb }}\left[A_{z}, A_{\bar{z}}\right] \\
=\overline{\mathcal{N}} \mathcal{N}_{\text {orb }} \int D \mu(\varphi) \exp \left\{2 \int _ { 0 } ^ { 1 / 2 } d t \int _ { \Sigma } d ^ { 2 } z \frac { \partial } { \partial t } \left[\left(\frac{k}{8 \pi}\left(A_{\bar{z}} A_{z}+\partial_{\bar{z}} \varphi \partial_{z} \varphi\right)-J^{0} \varphi\right)\right.\right. \\
\left.\left.\quad \times\left(f_{0}+f_{1}\right)-\frac{k}{4 \pi}\left(A_{z} \partial_{z} \varphi f_{0}-A_{\bar{z}} \partial_{\bar{z}} \varphi f_{1}\right)\right]\right\},
\end{aligned}
$$

where we have naturally defined two new orbifold wavefunctions at times $t=0$ and $t=1 / 2$ by

$$
\begin{aligned}
& \overline{\Psi_{0}^{\text {orb }}\left[A_{z}, A_{\bar{z}}\right]}=\overline{\mathcal{N}} \int D \mu\left(\varphi_{0}\right) \exp \left\{-\frac{k}{4 \pi} \int_{\Sigma} d^{2} z\left[A_{\bar{z}} A_{z}-2 A_{\bar{z}} \partial_{z} \varphi_{0}+\partial_{\bar{z}} \varphi_{0} \partial_{z} \varphi_{0}\right]\right\}, \\
& \Psi_{\frac{1}{2}}^{\text {orb }}\left[A_{z}, A_{\bar{z}}\right]=\mathcal{N}_{\text {orb }} \int D \mu\left(\varphi_{\frac{1}{2}}\right) \exp \left\{\frac{k f_{0}\left(\frac{1}{2}\right)}{2 \pi} \int_{\Sigma^{O}} d^{2} z\left[A_{z} \partial_{\bar{z}} \varphi_{\frac{1}{2}}-A_{\bar{z}} \partial_{z} \varphi_{\frac{1}{2}}\right]\right\},
\end{aligned}
$$

with $\Sigma^{O}=\Sigma / \mathbb{Z}_{2}[O]$ and $\mathbb{Z}_{2}[O]$ the cyclic group generated by the order two symmetry $O=\Omega$ or $O=\Omega C$ that we have chosen to quotient the theory by. Here and in the following we set the external charge density $J^{0}=0$ on the $t=0$ surface $\Sigma_{0}$. We have also introduced a new normalization constant $\mathcal{N}_{\text {orb }}$ for the wavefunction at $t=1 / 2$, which will be computed later on from the original normalization $\mathcal{N}$.

Let us now consider the orbifold path integral

$$
\begin{aligned}
Z_{\text {orb }} & =\left\langle\Psi_{0}^{\text {orb }} \mid \Psi_{\frac{1}{2}}^{\text {orb }}\right\rangle \\
& =\int D \mu_{G}\left(A_{z}, A_{\bar{z}}\right) \mathrm{e}^{2 i S_{\mathrm{TMGT}}^{\text {orb }}[A]} \overline{\Psi_{0}^{\text {orb }}\left[A_{z}, A_{\bar{z}}\right]} \Psi_{\frac{1}{2}}^{\text {orb }}\left[A_{z}, A_{\bar{z}}\right]
\end{aligned}
$$

where $S_{\mathrm{TMGT}}^{\mathrm{orb}}[A]$ is the topologically massive gauge theory action (2.2) for the orbifold of the three-dimensional theory, and the orbifold wavefunctions are given by (5.8). Here we have identified the actions 45

$$
2 S_{\mathrm{TMGT}}^{\mathrm{orb}}\left[A^{\mathrm{orb}}\right]=S_{\mathrm{TMGT}}[A]
$$

with $A$ regarded as the extension of the gauge field $A^{\text {orb }}$ from $([0,1] \times \Sigma) / \mathbb{Z}_{2}$ to the covering cylinder. To ease notation in the following we will not explicitly write the superscript orb on the orbifold fields. In the bulk of the worldsheet $\Sigma^{O}$ (neglecting boundary contributions over $\partial \Sigma^{O}$ ), this orbifold quantum field theory is gauge invariant. Under the bulk transformation (4.17) of the action, one may check explicitly that it is enough to shift the auxilliary 
field $\varphi$ on the two boundaries as

$$
\begin{aligned}
\varphi_{0} & \longmapsto \varphi_{0}-\Lambda, \\
\varphi_{\frac{1}{2}} & \longmapsto \varphi_{\frac{1}{2}}-\frac{\Lambda}{2 f_{0}\left(\frac{1}{2}\right)}
\end{aligned}
$$

in order to restore the gauge independence of all the factors under a bulk gauge transformation. What is particularly interesting here is that the wavefunction $\Psi_{\frac{1}{2}}^{\text {orb }}\left[A_{z}, A_{\bar{z}}\right]$ which emerges at the orbifold fixed point yields exactly the factor necessary to cancel the gauge dependent contribution coming from (4.17).

We can also rewrite the wavefunctions in terms of the normal and parallel components defined in section 3 to get

$$
\Psi_{\frac{1}{2}}^{\mathrm{orb}}\left[A_{z}, A_{\bar{z}}\right]=\mathcal{N}_{\text {orb }} \int D \mu(\varphi) \exp \left\{\frac{i k f_{0}\left(\frac{1}{2}\right)}{\pi} \int_{\Sigma^{O}} d^{2} z\left[A_{\|} \partial_{\perp} \varphi-A_{\perp} \partial_{\|} \varphi\right]\right\}
$$

Looking back at (3.3) we see a naive indication that the $\Omega T$ orbifold, containing only the term $\partial_{\|} \varphi$ at the boundary, imposes Dirichlet boundary conditions, while the $\Omega C T$ orbifold, for which only the factor with $\partial_{\perp} \varphi$ is present at the boundary, imposes Neumann boundary conditions. We will derive these results formally in the next subsection. Therefore, the emergence of the wavefunction $\Psi_{\frac{1}{2}}^{\mathrm{orb}}\left[A_{z}, A_{\bar{z}}\right]$ correctly imposes the anticipated boundary conditions at the orbifold line. In the next subsection we will also see that this wavefunction corresponds to the identity operator of the boundary conformal field theory. Note that in the cases where $\partial \Sigma^{O}=\emptyset$, we would get instead twisted and untwisted closed string sectors for the $\Omega T$ and $\Omega C T$ orbifolds, respectively, as studied in 33 .

However, as mentioned before, this is not the whole story, because if $\Sigma^{O}$ has a boundary, then the gauge fixed path integral acquires a gauge dependent piece and is given explicitly by

$$
\begin{aligned}
Z_{\text {orb }}= & \sqrt{\frac{\operatorname{det}^{\prime} \nabla_{\Sigma O}^{2}}{\mathcal{A}_{\Sigma^{O}}}} \int D \bar{A}_{z} D \bar{A}_{\bar{z}} \mathrm{e}^{2 i S_{\mathrm{TMGT}}^{\mathrm{orb}}[\bar{A}]} \overline{\Psi_{0}^{\text {orb }}\left[\bar{A}_{z}, \bar{A}_{\bar{z}}\right]} \Psi_{\frac{1}{2}}^{\mathrm{orb}}\left[\bar{A}_{z}, \bar{A}_{\bar{z}}\right] \\
& \times \iint D \mu(\Lambda) D \mu(\tilde{\varphi}) \delta(G(A)) \operatorname{det}\left[\frac{\delta G(A)}{\delta \Lambda}\right] \exp \left\{\frac{i k f_{0}\left(\frac{1}{2}\right)}{\pi} \oint_{\partial \Sigma^{O}} \Lambda \partial_{\perp} \tilde{\varphi}\right\}
\end{aligned}
$$

where $\tilde{\varphi}$ is the restriction of the auxilliary field $\varphi_{\frac{1}{2}}$ to the boundary $\partial \Sigma^{O}$. We now need to analyse carefully the orbifold identifications and check compatibility. For this, we consider the Hodge decompositions of the gauge fields $A_{i}$ at each time slice, which in the holomorphic polarization read

$$
\begin{aligned}
& A_{z}=a_{z}+\partial_{z} \bar{\phi} \\
& A_{\bar{z}}=a_{\bar{z}}+\partial_{\bar{z}} \phi
\end{aligned}
$$


where

$$
\phi=\phi_{R}+i \phi_{I}
$$

is a complex scalar field and $a_{i}$ is a harmonic one-form with

$$
\partial_{z} a_{\bar{z}} \pm \partial_{\bar{z}} a_{z}=0 .
$$

The harmonic one-forms have quantized periods $a / 2 \pi \in H^{1}(\Sigma, \mathbb{Z})$. Extending the identifications (3.3) on $\Sigma_{\frac{1}{2}}=\Sigma^{O}$ to the degrees of freedom $a_{i}$ and $\phi$ and to the gauge parameter $\Lambda$ one gets:

$$
\begin{array}{cccc}
\multicolumn{2}{c}{\boldsymbol{c} \Omega \mathbf{C T}} \\
\underline{\Sigma^{\Omega}} & \underline{\partial \Sigma^{\Omega}} & \underline{\sum^{\Omega C}} & \underline{\partial \Sigma^{\Omega C}} \\
a_{z} \equiv a_{\bar{z}} & a_{z} \in \mathbb{R} & a_{z} \equiv-a_{\bar{z}} & a_{z} \in i \mathbb{R} \\
a_{\|} \equiv-a_{\|} & a_{\|}=0 & a_{\|} \equiv a_{\|} & a_{\|} \neq 0 \\
a_{\perp} \equiv a_{\perp} & a_{\perp} \neq 0 & a_{\perp} \equiv-a_{\perp} & a_{\perp}=0 \\
\phi_{R} \equiv-\phi_{R} & \phi_{R}=0 & \phi_{R} \equiv \phi_{R} & \phi_{R} \neq 0 \\
\phi_{I} \equiv \phi_{I} & \phi_{I} \neq 0 & \phi_{I} \equiv-\phi_{I} & \phi_{I}=0 \\
\Lambda \equiv-\Lambda & \Lambda=0 & \Lambda \equiv \Lambda & \Lambda \neq 0 .
\end{array}
$$

\subsection{Boundary Conditions}

To analyse the partition function (5.13), we need to first specify a gauge condition. For this, we note that a gauge transformation only shifts the field $\phi_{R} \mapsto \phi_{R}+\Lambda$. So a natural choice is to impose the gauge constraint

$$
G(A)=\phi+\bar{\phi}=0
$$

so that the gauge fixed fields are

$$
\begin{aligned}
& \bar{A}_{z}=a_{z}-i \partial_{z} \phi_{I}, \\
& \bar{A}_{\bar{z}}=a_{\bar{z}}+i \partial_{\bar{z}} \phi_{I}
\end{aligned}
$$

while the scalar field $\phi_{R}$ has been integrated out and is no longer present in the path integral. Substituting this decomposition into the orbifold wavefunction $\Psi_{\frac{1}{2}}^{\text {orb }}$ at the new boundary at $t=1 / 2$ yields

$$
\Psi_{\frac{1}{2}}^{\text {orb }}\left[\bar{A}_{z}, \bar{A}_{\bar{z}}\right]=\mathcal{N}_{\text {orb }} \int D \mu(\varphi) \exp \left\{\frac{i k f_{0}\left(\frac{1}{2}\right)}{2 \pi} \int_{\Sigma^{O}} d^{2} z\left[\epsilon^{i j} a_{i} \partial_{j} \varphi+2 \sqrt{h} h^{i j} \partial_{i} \phi_{I} \partial_{j} \varphi\right]\right\} .
$$


Integrating by parts and using the fact that $a_{i}$ is a harmonic form then gives the path integral

$$
\begin{aligned}
Z_{\text {orb }}= & \sqrt{\frac{\operatorname{det}^{\prime} \nabla_{\Sigma^{O}}^{2}}{\mathcal{A}_{\Sigma^{O}}}} \mathcal{N}_{\text {orb }} \int D \bar{A}_{z} D \bar{A}_{\bar{z}} \mathrm{e}^{2 i S_{\mathrm{TMGT}}^{\mathrm{orb}}[\bar{A}]} \overline{\Psi_{0}^{\mathrm{orb}}\left[\bar{A}_{z}, \bar{A}_{\bar{z}}\right]} \\
& \times \iint D \mu(\Lambda) D \mu(\varphi) \exp \left\{\frac{i k f_{0}\left(\frac{1}{2}\right)}{\pi} \int_{\Sigma^{O}} d^{2} z \phi_{I} \nabla_{\Sigma^{O}}^{2} \varphi\right. \\
& \left.+i \oint_{\partial \Sigma^{O}}\left[\frac{k f_{0}\left(\frac{1}{2}\right)}{\pi}\left(a_{\perp} \varphi+\Lambda \partial_{\perp} \varphi+\phi_{I} \partial_{\|} \varphi\right)-2 \phi_{I} J^{\|}\right]\right\},
\end{aligned}
$$

where the measure for functional integration over the gauge fields is given by

$$
D \bar{A}_{z} D \bar{A}_{\bar{z}}=D a_{z} D a_{\bar{z}} D \mu\left(\phi_{I}\right) \sqrt{\frac{\operatorname{det}^{\prime} \nabla_{\Sigma}^{2}}{\mathcal{A}_{\Sigma}}} .
$$

The boundary source term in (5.21) arises from the minimal coupling in the action upon integrating by parts, and using the continuity equation (2.3) along with charge conservation on closed surfaces. With our choice of time-dependence for the conserved currents $J^{\mu}$, this is the only place that source terms remain.

One sees immediately that the integration over $\phi_{I}$ yields the Dirac delta-function constraint $\nabla_{\Sigma^{\circ}}^{2} \varphi=0$ in the bulk of $\Sigma^{O}$. This is expected because it corresponds to the equation of motion of the free chiral boson in the boundary conformal field theory. When $\partial \Sigma^{O}=\emptyset$, this constraint is the only remnant of the insertion of the wavefunction $\Psi_{\frac{1}{2}}^{\text {orb }}$. For the cases where $\partial \Sigma^{O} \neq \emptyset$, there are two types of wavefunctions $\Psi_{\frac{1}{2}}^{\text {orb }}$ corresponding to the two kinds of orbifolds under $\Omega T$ and $\Omega C T$. As we will now explicitly demonstrate, they correspond to the two possible conformal boundary conditions, Dirichlet and Neumann. More precisely, we will show that the orbifold path integral contains Dirac delta-functions correspondingly as:

$$
\begin{aligned}
\text { Dirichlet }(\Omega T) & : \delta\left(\partial_{\|} \varphi\right) \delta(\varphi-Y), \\
\text { Neumann }(\Omega C T) & : \delta\left(\partial_{\perp} \varphi\right),
\end{aligned}
$$

where $Y$ is some fixed function on $\partial \Sigma^{O}$. To do so, we must carefully analyse the field configurations compatible with the identifications in (5.17).

For the $\Omega T$ quotient, $a_{\perp} \neq 0, \Lambda=0$ and $\phi_{I} \neq 0$, and so the integrations over both $\phi_{I}$ and $a_{\perp}$ on $\partial \Sigma^{\Omega}$ are present and yield the respective Dirac delta-function constraints $\partial_{\|} \varphi=2 \pi J^{\|} / k f_{0}\left(\frac{1}{2}\right)$ and $\varphi=0$. These two conditions are compatible with each other and correspond to Dirichlet boundary conditions, the first constraint specifying that the field $\varphi$ is fixed at the boundary and the second one specifying what function it is fixed to. Indeed, by fixing the components of the external current parallel to the boundary line $\partial \Sigma^{O}$ to be

$$
J^{\|}=\frac{k f_{0}\left(\frac{1}{2}\right)}{2 \pi} \partial_{\|} Y,
$$


we can shift the boundary field $\varphi \mapsto \varphi+Y$, and rewrite the conditions as $\partial_{\|} \varphi=0$ and $\varphi=Y$. The value of the function on $\partial \Sigma^{O}$ can thereby be fixed to any desired one by the appropriate choice of external sources. The function $Y$ appearing in (5.24) may in fact be interpreted as the collective coordinate of a D-brane, and hence the minimal coupling to conserved currents allows the construction of D-branes in topological membrane theory [59]. Note that in this case the path integral does not depend on the gauge parameter $\Lambda$, so that these types of orbifolds do not break the gauge symmetries.

For the $\Omega C T$ orbifolds, $a_{\perp}=0, \Lambda \neq 0$ and $\phi_{I}=0$, and so only the integration over $\Lambda$ on $\partial \Sigma^{\Omega C}$ is present, yielding the Dirac delta-function constraint $\partial_{\perp} \varphi=0$. It corresponds to Neumann boundary conditions. Alternatively, we can think of this requirement as stating that the only boundary conditions compatible with these types of orbifolds are Neumann boundary conditions, because they are the only ones which are gauge invariant. This constraint could also have been deduced by using the Gauss law constraint (2.6) over $\partial \Sigma^{\Omega C}$ to solve for the orbifold boundary dependence of the wavefunctionals analogously to the construction of section 4 . The key identity which allows for this is the zero-mode equation (4.9) satisfied by the vacuum wavefunctionals (4.11). In this way it is possible to deal, in a completely analogous way, with the functional equation for the normal and longitudinal components on $\partial \Sigma^{\Omega C}$, and one easily checks that the gauge invariant solution thereby selects the value

$$
f_{0}\left(\frac{1}{2}\right)=\frac{1}{2}
$$

\subsection{Normalizations}

The main conclusion of the previous subsection is that the insertion of the orbifold wavefunction at $t=1 / 2$ merely serves to constrain the bulk dynamics of the WZNW field $\varphi$ on $\Sigma^{O}$ with the appropriate boundary conditions. After integration over $\varphi$, there is no further field dependence from this boundary, and the quantum state on $\Sigma^{O}$ is formally unity $|1\rangle$, in agreement with our earlier expectations. Similarly, integrating out the local parts $\varphi$ and $\phi_{I}$ of the wavefunction at $t=0$ produces some overall determinant factors, along with the integrations over the harmonic forms $a_{i}$. The latter quantities transform under large gauge transformations, thereby encoding the topological nature of the solutions and the correspondence between the wavefunctions and conformal blocks. They will be dealt with in the next section. In this subsection we will carefully tabulate the determinant factors that arise from integration over the local components of the fields. They will incorporate part of the moduli dependence of the resulting states.

The key ingredient in the computation is the fixing of the normalization constant $\mathcal{N}$. The canonical choice is to demand that the norms of an orthogonal basis of wavefunctions 
in the unorbifolded theory be equal to unity, implemented by the functional integration

$$
1=\int D \mu_{G}\left(A_{z}, A_{\bar{z}}\right) \overline{\Psi_{0}\left[A_{z}, A_{\bar{z}}\right]} \Psi_{0}\left[A_{z}, A_{\bar{z}}\right]
$$

The corresponding partition function is then a vacuum-to-vacuum amplitude and it leads to a well-defined inner product on the quantum Hilbert space of physical states. The way to correctly define this object is by identifying (gluing) the two boundaries $\Sigma_{0}$ and $\Sigma_{1}$. The explicit computation can be found in [31,34] while the correct relative boundary conditions are given in 23, 32]. One finds that the fields $\phi_{I}$ coming from the Hodge decomposition of the gauge field must be identified at the two boundaries at $t=0$ and $t=1$, while the boundary conformal field theory degrees of freedom are combined into the single scalar field $\varphi_{d}=\varphi_{0}-\varphi_{1}$. Note that while $\varphi_{0}$ and $\varphi_{1}$ are chiral worldsheet fields, their difference $\varphi_{d}$ is not. The Lagrangian for the field $\varphi_{d}$ is proportional to $k \partial_{z} \varphi_{d} \partial_{\bar{z}} \varphi_{d}$ and its functional integration measure is $D \mu\left(\varphi_{d}\right)$. The path integral over this field thereby produces precisely the same determinant factor that appears in (4.13), times a factor $\sqrt{k}$ involving the ChernSimons coefficient. The integration over the gauge degree of freedom $\phi_{I}$ produces no such determinant factors and yields the overall constant $\sqrt{k}$, because its functional integration measure is $D\left(\partial_{z} \phi_{I}\right) D\left(\partial_{\bar{z}} \phi_{I}\right)$ and it only appears in the path integral through its worldsheet derivatives. As mentioned at the beginning of this section, the same is true of the integration over the gauge parameters $\Lambda$. Thus, to properly normalize the wavefunctionals of section 4 , we will take the normalization constant

$$
\mathcal{N}=\left(\frac{\operatorname{det}^{\prime} \nabla_{\Sigma}^{2}}{k^{2} \mathcal{A}_{\Sigma}}\right)^{1 / 4}
$$

Integrating out all the local fields in the path integral (4.21) in this way produces an effective inner product $(\cdot, \cdot)_{a}$ depending solely on the harmonic gauge fields $a_{i}$ on the two boundaries $\Sigma_{0}$ and $\Sigma_{1}$. This inner product will be worked out explicitly in the next section. Incorporating the normalization factor (5.27) into (4.21) we then arrive at

$$
Z_{\Psi}=\sqrt{\frac{\mathcal{A}_{\Sigma}}{\operatorname{det}^{\prime} \nabla_{\Sigma}^{2}}}\left(\Psi_{0}, \Psi_{1}\right)_{a} .
$$

In (5.28) the wavefunctions are obtained by integrating out all local field dependences.

We can now easily work out the normalization constant $\mathcal{N}_{\text {orb }}$ of the orbifold wavefunctions from the un-orbifolded one (5.27). The crucial observation is that $\mathcal{N}$ is defined through the path integral with action $k \int_{\Sigma} d^{2} z \partial_{z} \varphi_{d} \partial_{\bar{z}} \varphi_{d}$. Under the orbifold operations the integrand of this action is invariant but the orientation of the worldsheet $\Sigma$ reverses due to the transformation $z \mapsto-\bar{z}$, i.e. this action vanishes on the resulting orbifolded surface $\Sigma^{O}$. Note that this fact was used already in the construction of the orbifold wavefunction 
at $t=1 / 2$ in (5.8). In other words, the determinant of the scalar Laplacian on $\Sigma^{O}$ with the given orbifold boundary conditions is equal to unity. We thereby conclude that

$$
\mathcal{N}_{\text {orb }}=1
$$

Completely analogous arguments establish that the functional integrals over $\phi_{I}$ and $\Lambda$ at $t=1 / 2$ contribute only the usual $\sqrt{k}$ constants to the orbifold partition function (5.21). The only non-trivial local field dependence that remains comes from the integral over the $t=0$ wavefunction, through its normalization constant (5.27) and its dependence on $\varphi_{0}$ which yields the determinant factor in (4.13). Remembering that the wavefunction at $t=1 / 2$ is unity, the orbifold path integral is thereby given through the properly normalized topological inner product

$$
Z_{\mathrm{orb}}=\left(\frac{\mathcal{A}_{\Sigma}}{\operatorname{det}^{\prime} \nabla_{\Sigma}^{2}}\right)^{1 / 4}\left(\Psi_{0}^{\mathrm{orb}}, 1\right)_{a}
$$

Note that the orbifold state $\overline{\Psi_{0}^{\text {orb }}}$ corresponding to $\Sigma_{0}$ is identical to that in $(4.15)$, except that the argument of its exponential is twice as large. Again, in (5.30) it is understood that the wavefunction depends only on the harmonic one-forms $a_{i}$.

\section{Character Expansions}

In this section we will finally come to the main objective of this paper. We will complete the evaluation of the orbifold partition functions of the previous section, using them to construct modular invariants for the induced conformal field theories. For this, we will evaluate the path integrals over the harmonic components $a_{i}$ of the gauge fields, assuming that the the external currents $J^{\mu}$ are cohomologically trivial, as is appropriate for the vacuum amplitudes. As an explicit example, we will look at various toroidal orbifolds and show that the topological membrane formalism yields the anticipated results in these cases.

\subsection{Effective Topological Wavefunctions}

We now proceed to determine the dependence of the wavefunctions on the harmonic forms $a_{i}$. For this, we work in a holomorphic polarization, with respect to the chosen complex structure of $\Sigma$ which we take to be of genus $g$, and decompose the harmonic forms into the sums 28

$$
\begin{aligned}
& a_{z}(\bar{z})=\pi i \sum_{\ell=1}^{g} a^{\ell}\left(\Gamma_{2}^{-1}\right)^{\ell \ell^{\prime}} \overline{\omega_{\ell^{\prime}}(z)} \\
& a_{\bar{z}}(z)=-\pi i \sum_{\ell=1}^{g} \bar{a}^{\ell}\left(\Gamma_{2}^{-1}\right)^{\ell \ell^{\prime}} \omega_{\ell^{\prime}}(z)
\end{aligned}
$$


where $\omega_{\ell}=\omega_{\ell}(z) d z, \ell=1, \ldots, g$ form a basis of holomorphic one-differentials determined by the conditions

$$
\begin{aligned}
& \oint_{\alpha_{\ell}} \omega_{\ell^{\prime}}=\delta_{\ell^{\prime}}^{\ell}, \\
& \oint_{\beta^{\ell}} \omega_{\ell^{\prime}}=\Gamma_{\ell \ell^{\prime}} .
\end{aligned}
$$

Here $\alpha_{\ell}, \beta^{\ell}, \ell=1, \ldots, g$ form a canonical set of closed contours on the Riemann surface $\Sigma$ which generate its first homology group $H_{1}(\Sigma, \mathbb{Z})$. The $g \times g$ complex period matrix $\Gamma$ of the surface is symmetric, and we will decompose it into real and imaginary parts as $\Gamma=\Gamma_{1}+i \Gamma_{2}$. Its imaginary part $\Gamma_{2}$ is a positive definite symmetric matrix. The $\omega_{\ell}$ span the kernel of the antiholomorphic derivative $\partial_{\bar{z}}$ and are normalized as

$$
\int_{\Sigma} d^{2} z \omega_{\ell}(z) \overline{\omega_{\ell^{\prime}}(z)}=-2 i\left(\Gamma_{2}\right)_{\ell \ell^{\prime}}
$$

The gauge transformations $\Sigma \rightarrow U(1)$ of the theory have integer winding numbers around homologically non-trivial curves in $\Sigma$. A map which winds $s^{\ell}$ times around the primitive cycle $\alpha_{\ell}$ and $r_{\ell}$ times around $\beta^{\ell}$ determines a large gauge transformation $]$

$$
\begin{aligned}
& a^{\ell} \longmapsto a^{\ell}+s^{\ell}+\Gamma^{\ell \ell^{\prime}} r_{\ell^{\prime}}, \\
& \bar{a}^{\ell} \longmapsto \bar{a}^{\ell}+s^{\ell}+\bar{\Gamma}^{\ell \ell^{\prime}} r_{\ell^{\prime}} .
\end{aligned}
$$

The topological dependence of the Schrödinger wavefunctionals may now be uniquely fixed by requiring that they furnish a projective representation of the large gauge symmetries. Let us consider a rational Chern-Simons coefficient $k=2 p / q$, with $p$ even. Then the projective representation defines a semi-periodicity condition on the wavefunctions which is solved by functions that live in a finite-dimensional vector space. With the holomorphic parametrization above, we can build an orthogonal basis, with $(p q)^{g}$ members $\left((2 p q)^{g}\right.$ members for $p$ odd), for the wavefunctions in the form [28, 40 42]

$$
\Psi_{\lambda}(a, \bar{a} ; \Gamma)=\exp \left\{\frac{k \pi}{8} a^{\ell}\left(\Gamma_{2}^{-1}\right)_{\ell \ell^{\prime}}\left(a^{\ell^{\prime}}-\bar{a}^{\ell^{\prime}}\right)\right\} \Theta_{p}\left[\begin{array}{c}
\frac{\lambda}{q} \\
0
\end{array}\right]\left(\sqrt{2} a \mid \frac{2 \Gamma}{k}\right),
$$

where $\lambda$ is an integer $g$-vector with components in the range $\lambda^{\ell}=0,1, \ldots, p q-1$. The genus $g$ modified Jacobi theta-functions are defined by

$$
\begin{aligned}
& \Theta_{p}\left[\begin{array}{l}
\frac{\lambda}{q} \\
0
\end{array}\right]\left(\sqrt{2} a \mid \frac{2 \Gamma}{k}\right) \\
& \quad=\sum_{s \in p \mathbb{Z}^{g}} \exp \left\{\frac{2 \pi i}{k}\left(s^{\ell}+\frac{\lambda^{\ell}}{q}\right) \Gamma_{\ell \ell^{\prime}}\left(s^{\ell^{\prime}}+\frac{\lambda^{\ell^{\prime}}}{q}\right)+2 \sqrt{2} \pi i a_{l}\left(s^{\ell}+\frac{\lambda^{\ell}}{q}\right)\right\},
\end{aligned}
$$

\footnotetext{
${ }^{3}$ Here and in the following the topological indices $\ell$ are raised and lowered with the metric $G_{\ell \ell^{\prime}}=2\left(\Gamma_{2}\right)_{\ell \ell^{\prime}}$ on the space of holomorphic harmonic one-forms.
} 
where by "modified" we mean that the integer sums are restricted to multiples of $p$.

The wavefunctions (6.5) are those for the first Landau level on the Riemann surface $\Sigma$. They carry a $(p q)^{g}$-dimensional unitary irreducible representation of the holonomy algebra of the gauge theory in terms of clock and cyclic shift operators as

$$
\begin{aligned}
& \exp \left\{i \oint_{\alpha_{\ell}} A\right\} \Psi_{\lambda}(a, \bar{a} ; \Gamma)=\mathrm{e}^{2 \pi i \lambda^{\ell} / q} \Psi_{\lambda}(a, \bar{a} ; \Gamma), \\
& \exp \left\{i \oint_{\beta^{\ell}} A\right\} \Psi_{\lambda}(a, \bar{a} ; \Gamma)=\Psi_{\lambda+q 1_{\ell}(\bmod p q)}(a, \bar{a} ; \Gamma),
\end{aligned}
$$

where $1_{\ell}$ is the integer $g$-vector with components $\left(1_{\ell}\right)^{\ell^{\prime}}=\delta_{\ell}^{\ell^{\prime}}$. As described in section 2.2. the representation (6.7) explicitly realizes the monopole processes which change the charge of the quantum states. The normalization of the Chern-Simons coefficient, which differs from the standard ones [41], is chosen so that the $U(1)$ charges carried by Wilson lines belong to the required spectrum (2.16) of the theory. In this way, the large gauge transformations (6.4) are restricted to those with $s^{\ell}, r_{\ell}=0 \bmod p$. Note that $\lambda^{\ell} / q$ are primary charges and that for the rational values $k=2 p / q$ there is one charge independent monopole process with $\Delta Q=p$. In terms of the induced conformal field theory these shifts of charge lie within a single family [32]. Then the restricted large gauge transformations (6.4) are just the allowed monopole processes which shift charges inside the same family or conformal block.

Let us now consider the unorbifolded path integral over the topological components of the gauge fields in (5.28). As we did in section 5.3, we will start by fixing the normalization of the effective wavefunctions. For this, we define an inner product between wavefunctions $\Psi_{\lambda}(a, \bar{a} ; \Gamma)$ and $\Psi_{\lambda^{\prime}}(a, \bar{a} ; \Gamma)$ by identifying the $a_{i}$ fields on the boundaries $\Sigma_{0}$ and $\Sigma_{1}$ and integrating out their topological dependence as

$$
\left(\Psi_{\lambda}, \Psi_{\lambda^{\prime}}\right)_{a}=\frac{1}{2^{g} \operatorname{det} \Gamma_{2}} \int_{\mathcal{J}(\Sigma)} \prod_{\ell=1}^{g} d a^{\ell} d \bar{a}^{\ell} \overline{\Psi_{\lambda}(a, \bar{a} ; \Gamma)} \Psi_{\lambda^{\prime}}(a, \bar{a} ; \Gamma)
$$

where, due to the large gauge invariance of this inner product, the integration is restricted to the Jacobian variety $\mathcal{J}(\Sigma)$ of the Riemann surface $\Sigma$ obtained by reducing the $a, \bar{a}$ phase space to the plaquette of gauge orbits

$$
\begin{aligned}
& a^{\ell}=\rho^{\ell}+\Gamma^{\ell \ell^{\prime}} \gamma_{\ell^{\prime}}, \\
& \bar{a}^{\ell}=\rho^{\ell}+\bar{\Gamma}^{\ell \ell^{\prime}} \gamma_{\ell^{\prime}}
\end{aligned}
$$

with $\rho^{\ell}, \gamma_{\ell} \in[0, p]$. This is the inner product that is inherited from the normalization integral of (5.26), and also that which naturally arises in the geometric quantization of pure Chern-Simons gauge theory. 
As explained in section 2.2, one must supplement this vacuum amplitude with monopole transitions enforcing large gauge invariance, and so we compute instead

$$
\begin{aligned}
\left(\Psi_{\lambda},\right. & \left.\exp \left\{-i \sum_{\ell=1}^{g} \Delta Q_{\lambda}^{\ell} \oint_{\beta^{\ell}} A\right\} \Psi_{\lambda^{\prime}}\right)_{a} \\
= & \sum_{s \in p \mathbb{Z}^{g}} \sum_{s^{\prime} \in p \mathbb{Z}^{g}} \prod_{\ell=1}^{g} \int_{0}^{p} d \rho^{\ell} \exp \left\{2 \sqrt{2} \pi i \rho_{\ell}\left(s^{\prime \ell}-s^{\ell}+\frac{\lambda^{\prime \ell}}{q}-\frac{\lambda^{\ell}}{q}-\Delta Q_{\lambda}^{\ell}\right)\right\} \\
& \times \prod_{\ell=1}^{g} \int_{0}^{p} d \gamma_{\ell} \exp \left\{-\frac{k \pi}{2} \gamma_{\ell}\left(\Gamma_{2}\right)^{\ell^{\prime}} \gamma_{\ell^{\prime}}+2 \sqrt{2} \pi i \gamma^{\ell}\left[\Gamma_{\ell \ell^{\prime}}\left(s^{\ell^{\prime}}+\frac{\lambda^{\prime \ell^{\prime}}}{q}\right)\right.\right. \\
& \left.-\bar{\Gamma}_{\ell \ell^{\prime}}\left(s^{\ell^{\prime}}+\frac{\lambda^{\ell^{\prime}}}{q}+\Delta Q_{\lambda}^{\ell^{\prime}}\right)\right]+\frac{2 \pi i}{k}\left[\left(s^{\ell \ell}+\frac{\lambda^{\prime \ell}}{q}\right) \Gamma_{\ell \ell^{\prime}}\left(s^{\prime \ell^{\prime}}+\frac{\lambda^{\ell^{\prime}}}{q}\right)\right. \\
& \left.\left.-\left(s^{\ell}+\frac{\lambda^{\ell}}{q}+\Delta Q_{\lambda}^{\ell}\right) \bar{\Gamma}_{\ell \ell^{\prime}}\left(s^{\ell^{\prime}}+\frac{\lambda^{\ell^{\prime}}}{q}+\Delta Q_{\lambda}^{\ell^{\prime}}\right)\right]\right\} .
\end{aligned}
$$

The effect of the monopole charges $\Delta Q_{\lambda}^{\ell}$ is to shift a charge $Q_{\lambda}^{\ell}$ on one boundary to the charge $Q_{\bar{\lambda}}^{\ell}=Q_{\lambda}^{\ell}+\Delta Q_{\lambda}^{\ell}$ on the other boundary. Let us assume a decomposition $Q_{\lambda}^{\ell}=\lambda^{\ell} / q$ of the charge as in (2.20), where for each $\ell=1, \ldots, g$ the integers $\left(m^{\ell}, n^{\ell}\right)$ form a corresponding Bezout pair, i.e. the smallest integers satisfying this decomposition. Then, as discussed in section 2, the opposite charge $Q_{\bar{\lambda}}^{\ell}=\bar{\lambda}^{\ell} / q$ is given by (2.21) and therefore $\Delta Q_{\lambda}^{\ell}=-k n^{\ell} / 2$.

The integration over the variables $\rho^{\ell}$ in (6.10) thereby gives $2^{-g / 2} \delta_{\mathcal{J}(\Sigma)}^{(g)}\left(\frac{\lambda^{\prime}-\lambda}{q}-\frac{p n}{q}\right)$, where the periodic delta function, invariant under the large gauge transformations (6.4), on the complex $g$-torus $\mathcal{J}(\Sigma)$ is given by

$$
\delta_{\mathcal{J}(\Sigma)}^{(2 g)}(\mathbf{a})=\sum_{s \in p \mathbb{Z}^{g}} \sum_{r \in p \mathbb{Z}^{g}} \delta^{(2 g)}(\mathbf{a}+s+\Gamma \cdot r)
$$

This sets $\lambda^{\prime \ell} / q=\bar{\lambda}^{\ell} / q \bmod p$ for each $\ell=1, \ldots, g$. The sum over $s^{\prime} \in p \mathbb{Z}^{g}$ in (6.10) then enforces the $\bmod p$ periodicity in this identification, and upon completing the square in the resulting terms involving the $\gamma_{\ell}$ variables we get

$$
\begin{aligned}
\left(\Psi_{\lambda},\right. & \left.\exp \left\{-i \sum_{\ell=1}^{g} \Delta Q_{\lambda}^{\ell} \oint_{\beta^{\ell}} A\right\} \Psi_{\lambda^{\prime}}\right)_{a} \\
= & \frac{\delta_{\lambda^{\prime}, \bar{\lambda}}}{2^{g / 2}} \sum_{s \in p \mathbb{Z}^{g}} \prod_{\ell=1}^{g} d \gamma_{\ell} \exp \left\{-\frac{k \pi}{2}\left[\gamma^{\ell}+\frac{2 \sqrt{2}}{k}\left(s^{\ell}+\frac{\bar{\lambda}^{\ell}}{q}\right)\right]\left(\Gamma_{2}\right)_{\ell \ell^{\prime}}\right. \\
\times & {\left.\left[\gamma^{\ell^{\prime}}+\frac{2 \sqrt{2}}{k}\left(s^{\ell^{\prime}}+\frac{\bar{\lambda}^{\ell^{\prime}}}{q}\right)\right]\right\} . }
\end{aligned}
$$

Shifting variables $\gamma^{\ell} \mapsto \gamma^{\ell}-2 \sqrt{2}\left(s^{\ell}+\bar{\lambda}^{\ell} / q\right) / k$ in (6.12) and using the sum over $s \in p \mathbb{Z}^{g}$ to extend the integration domain from the gauge orbit space to all of $\mathbb{R}^{g}$ yields a Gaussian 
integral in the $\gamma_{\ell}$ 's, and we arrive finally at

$$
\left(\Psi_{\lambda}, \exp \left\{-i \sum_{\ell=1}^{g} \Delta Q_{\lambda}^{\ell} \oint_{\beta^{\ell}} A\right\} \Psi_{\lambda^{\prime}}\right)_{a}=\frac{1}{k^{g / 2} \sqrt{\operatorname{det} \Gamma_{2}}} \delta_{\lambda^{\prime}, \bar{\lambda}},
$$

where as before we drop all irrelevant numerical constants.

Using the normalizations $(5.28)$ and $(6.13)$, we can now write down the appropriate wavefunctions whose products will generate the desired partition function. After integrating out the bulk modes, the path integral (4.21) decomposes into two independent integrals over the modes associated with each of the two boundaries. We therefore define the total effective wavefunctions by integrating out their $a_{i}$ field dependences explicitly using the inner product (6.8), and we arrive at

$$
\begin{aligned}
\Psi_{\lambda}(\Gamma)= & \left(\frac{\mathcal{A}_{\Sigma}}{\operatorname{det}^{\prime}\left(\nabla_{\Sigma}^{2}\right) \operatorname{det}\left(k \Gamma_{2}\right)}\right)^{1 / 4}\left(1, \Psi_{\lambda}\right)_{a} \\
= & \left(\frac{\mathcal{A}_{\Sigma}}{\operatorname{det}^{\prime}\left(\nabla_{\Sigma}^{2}\right) \operatorname{det}\left(k \Gamma_{2}\right)}\right)^{1 / 4} \sum_{s \in p \mathbb{Z}^{g}} \prod_{\ell=1}^{g} \int_{0}^{p} \int_{0}^{p} d \rho^{\ell} d \gamma_{\ell} \exp \left\{2 \pi i\left(\rho^{\ell}+i\left(\Gamma_{2}\right)^{\ell \ell^{\prime}} \gamma_{\ell^{\prime}}\right)\right. \\
& \left.\times\left[\frac{k}{8} \gamma_{\ell}+\sqrt{2}\left(s_{\ell}+\frac{\lambda_{\ell}}{q}\right)\right]+\frac{2 \pi i}{k}\left(s^{\ell}+\frac{\lambda^{\ell}}{q}\right) \Gamma_{\ell \ell^{\prime}}\left(s^{\ell^{\prime}}+\frac{\lambda^{\ell^{\prime}}}{q}\right)\right\} \\
= & \left(\frac{\mathcal{A}_{\Sigma}}{\operatorname{det}^{\prime}\left(\nabla_{\Sigma}^{2}\right) \operatorname{det}\left(k \Gamma_{2}\right)}\right)^{1 / 4} \sum_{s \in p \mathbb{Z}^{g}} \exp \left\{\frac{2 \pi i}{k}\left(s^{\ell}+\frac{\lambda^{\ell}}{q}\right) \Gamma_{\ell \ell^{\prime}}\left(s^{\ell^{\prime}}+\frac{\lambda^{\ell^{\prime}}}{q}\right)\right\} .
\end{aligned}
$$

\subsection{Statistical Sums}

Up to a factor independent of the geometry of the Riemann surface $\Sigma$, the wavefunctions (6.14), along with the charge selection rule, naturally lead to the partition function for the rational conformal field theory of a compactified free scalar field. In string units the radius of compactification $R$ is given through the relation $k=2 R^{2}$, so that the conformal field theory partition function $Z_{\Sigma}$ is given by

$$
\begin{aligned}
Z_{\Sigma}= & k^{g / 2} Z_{\Psi} \\
= & k^{g / 2} \sum_{\lambda \in\left(\mathbb{Z}_{p q}\right)^{g}} \overline{\Psi_{\bar{\lambda}}(\Gamma)} \Psi_{\lambda}(\Gamma) \\
= & \sqrt{\frac{\mathcal{A}_{\Sigma}}{\operatorname{det}^{\prime}\left(\nabla_{\Sigma}^{2}\right) \operatorname{det} \Gamma_{2}}} \sum_{\lambda \in\left(\mathbb{Z}_{p q}\right)^{g}} \sum_{s \in p \mathbb{Z}^{g}} \sum_{s^{\prime} \in p \mathbb{Z}^{g}} \exp \left\{\frac{2 \pi i}{k}\left(s^{\ell}+\frac{\lambda^{\ell}}{q}\right) \Gamma_{\ell \ell^{\prime}}\left(s^{\ell^{\prime}}+\frac{\lambda^{\ell^{\prime}}}{q}\right)\right. \\
& \left.-\frac{2 \pi i}{k}\left(s^{\ell}+\frac{\bar{\lambda}^{\ell}}{q}\right) \bar{\Gamma}_{\ell \ell^{\prime}}\left(s^{\ell^{\prime}}+\frac{\bar{\lambda}^{\ell^{\prime}}}{q}\right)\right\} .
\end{aligned}
$$


It is now straightforward to compute the orbifold partition functions given the analysis of the previous subsection. As we have seen before, for the orbifold theories we have only the wavefunction at the boundary $\Sigma_{0}$. Moreover, the charge spectrum is appropriately truncated [32]. It is now clear in fact that the integers $m^{\ell}$ correspond to the Kaluza-Klein modes of the compactification while $n^{\ell}$ correspond to winding numbers. Thus not all of the wavefunctions $\Psi_{\lambda}$ obtained above will survive the orbifold operations. Precisely, only $(p q / 2)^{g}$ of them remain, so that the dimension of the physical state space is reduced by a factor of $2^{-g}$. Recall that $p$ is even, so that this counting of states is coherent. In the case that $p$ is odd the number of linearly independent states is reduced from $(2 p q)^{g}$ to $(p q)^{g}$ by the orbifold operations.

For the $\Omega C T$ symmetries the charge spectrum is truncated to the values

$$
\frac{\lambda^{\ell}}{q}=m^{\ell}, \quad m^{\ell}=0, \ldots, \frac{p q}{2}-1
$$

and the harmonic fields obey $a^{\ell}=-\bar{a}^{\ell}=i\left(\Gamma_{2}\right)^{\ell \ell^{\prime}} \gamma_{\ell^{\prime}}$. Furthermore, the period matrix is necessarily purely imaginary, $\Gamma=i \Gamma_{2}$, for compatibility with this orbifold. Following through the analysis of the previous subsection, in this case there are no $\rho^{\ell}$ integrations, and together with the doubling of the argument of the exponential factor in the wavefunction $\Psi_{\lambda}^{\text {orb }}$ we find an overall normalization factor $2^{-g}$, as expected.

On the other hand, for the $\Omega T$ symmetries the spectrum is truncated as

$$
\frac{\lambda^{\ell}}{q}=\frac{k}{4} n^{\ell}, \quad n^{\ell}=0, \ldots, \frac{p q}{2}-1
$$

and in this case $a^{\ell}=\bar{a}^{\ell}=\rho^{\ell}+\left(\Gamma_{1}\right)^{\ell \ell^{\prime}} \gamma_{\ell^{\prime}}$. There are now no Gaussian $\gamma_{\ell}$ integrations arising, and the gauge orbit integral over the new variables $\rho^{\ell}+\left(\Gamma_{1}\right)^{\ell^{\prime}} \gamma_{\ell^{\prime}}$ produces an extra multiplicative factor $2^{-g / 2}$. The overall normalization factor obtained in this case is therefore $2^{-g} \sqrt{\operatorname{det}\left(k \Gamma_{2}\right)}$.

Explicitly, we have thereby obtained the total orbifold partition functions

$$
\begin{aligned}
Z_{\Sigma^{O}} & =k^{g / 4} Z_{\text {orb }} \\
& =k^{g / 4} \sum_{\lambda \in\left(\mathbb{Z}_{\frac{p q}{2}}\right)^{g}} \Psi_{\lambda}^{\text {orb }}(\Gamma),
\end{aligned}
$$

which for the two types of orbifold constructions under consideration here are given by

$$
Z_{\Sigma^{\Omega C}}=\frac{1}{2^{g}}\left(\frac{\mathcal{A}_{\Sigma}}{\operatorname{det}^{\prime}\left(\nabla_{\Sigma}^{2}\right) \operatorname{det} \Gamma_{2}}\right)^{1 / 4} \sum_{m \in\left(\mathbb{Z}_{\frac{p q}{2}}\right)^{g}} \sum_{s \in p \mathbb{Z}^{g}} \exp \left\{\frac{2 \pi i}{k}\left(s^{\ell}+m^{\ell}\right) \Gamma_{\ell \ell^{\prime}}\left(s^{\ell^{\prime}}+m^{\ell^{\prime}}\right)\right\}
$$


and

$$
Z_{\Sigma^{\Omega}}=\frac{k^{g / 2}}{2^{g}}\left(\frac{\mathcal{A}_{\Sigma} \operatorname{det} \Gamma_{2}}{\operatorname{det}^{\prime} \nabla_{\Sigma}^{2}}\right)^{1 / 4} \sum_{n \in\left(\mathbb{Z}_{\frac{p q}{2}}\right)^{g}} \sum_{s \in p \mathbb{Z}^{g}} \exp \left\{\frac{2 \pi i}{k}\left(s^{\ell}+\frac{k}{4} n^{\ell}\right) \Gamma_{\ell \ell^{\prime}}\left(s^{\ell^{\prime}}+\frac{k}{4} n^{\ell^{\prime}}\right)\right\}
$$

These formulas all hold for arbitrary genus $g$. For $g=0$ there are no moduli and one obtains only trivial determinant factors. The case $g=1$ will be analysed in detail in the next subsection.

For genus $g \geq 2$, the formulas above are invariant under the natural action of the Siegal modular group $S p(2 g, \mathbb{Z})$ on the space of period matrices. They should be supplemented by the explicit expression for the determinant of the scalar Laplacian on the hyperbolic Riemann surface $\Sigma$ which is given by 65

$$
\begin{aligned}
\operatorname{det}^{\prime} \nabla_{\Sigma}^{2}= & \frac{\mathcal{A}_{\Sigma} \operatorname{det} \Gamma_{2}}{8 \pi^{2}} \mathrm{e}^{2 S_{\mathrm{L}}\left[h_{z \bar{z}}\right]}\left[\frac{\left[\Theta\left[\begin{array}{l}
0 \\
0
\end{array}\right]\left(\sum_{\ell=1}^{g} \int_{z_{0}}^{z_{\ell}} \omega-\int_{z_{0}}^{w} \omega-\triangle \mid \Gamma\right)\right.}{\operatorname{det}_{\ell, \ell^{\prime}}\left[\omega_{\ell}\left(z_{\ell^{\prime}}\right)\right] \sigma(w)}\right. \\
& \left.\times \prod_{\ell=1}^{g} \frac{\sigma\left(z_{\ell}\right)}{E\left(z_{\ell}, w\right)} \prod_{\ell^{\prime}<\ell} E\left(z_{\ell^{\prime}}, z_{\ell}\right)\right]^{4 / 3},
\end{aligned}
$$

where

$$
S_{\mathrm{L}}\left[h_{z \bar{z}}\right]=\frac{1}{48 \pi} \int_{\Sigma} d^{2} z \partial_{z} \ln \left(h_{z \bar{z}}\right) \partial_{\bar{z}} \ln \left(h_{z \bar{z}}\right)
$$

is the Liouville action in the conformal gauge, and $z_{1}, \ldots, z_{g}, w$ are arbitrary points on $\Sigma$. The ordinary Jacobi theta-functions are given by

$$
\Theta\left[\begin{array}{l}
c \\
d
\end{array}\right](u \mid \Gamma)=\sum_{s \in \mathbb{Z}^{g}} \exp \left\{\pi i\left(s^{\ell}+c^{\ell}\right) \Gamma_{\ell \ell^{\prime}}\left(s^{\ell^{\prime}}+c^{\ell^{\prime}}\right)+2 \pi i\left(s^{\ell}+c^{\ell}\right)\left(u_{\ell}+d_{\ell}\right)\right\}
$$

for $c^{\ell}, d_{\ell} \in[0,1]$ and $\mathbf{u} \in \mathbb{C}^{g}$, while

$$
\triangle_{\ell}=\frac{1}{2}-\frac{1}{2} \Gamma_{\ell \ell}+\sum_{\ell^{\prime} \neq \ell} \oint_{\alpha_{\ell^{\prime}}} d z \omega_{\ell^{\prime}}(z) \int_{z_{0}}^{z} \omega_{\ell}
$$

is the vector of Riemann constants which is related to the Riemann divisor class of $\Sigma$. The prime form is given by

$$
E\left(z, z_{0}\right)=\frac{\Theta\left[\begin{array}{l}
1 / 2 \\
1 / 2
\end{array}\right]\left(\int_{z_{0}}^{z} \omega \mid \Gamma\right)}{\sqrt{h_{E}(z) h_{E}\left(z_{0}\right)}}
$$


with the $\frac{1}{2}$-differentials

$$
h_{E}(z)=\left.\omega^{\ell}(z) \frac{\partial}{\partial u^{\ell}} \Theta\left[\begin{array}{l}
1 / 2 \\
1 / 2
\end{array}\right](u \mid \Gamma)\right|_{u=0} .
$$

The prime form is antisymmetric under interchange of its arguments and behaves like $z-z_{0}$ for $z \rightarrow z_{0}$. Finally, the quantities $\sigma(z)$ are twisted differentials of rank $g / 2$ which carry the conformal anomaly, and which may be defined by choosing a set of points $P_{1}, \ldots, P_{g}$ on $\Sigma$ and setting their ratios as

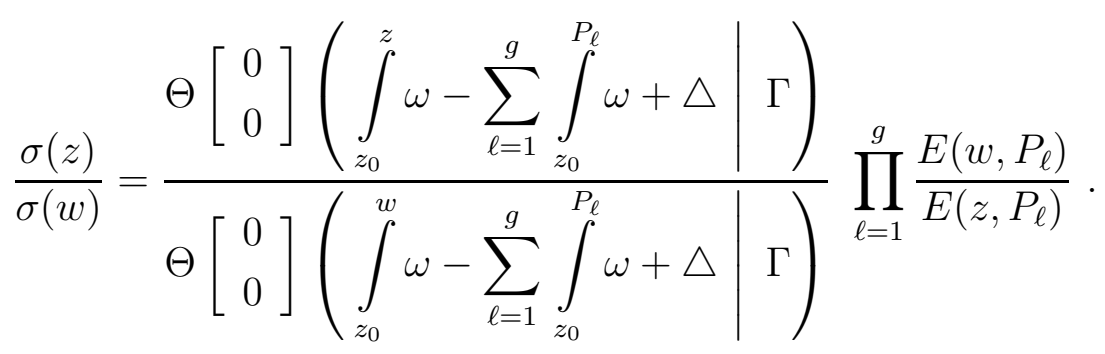

It follows from the Riemann vanishing theorem that the ratios (6.27) are independent of the choice of points $P_{\ell} \in \Sigma$, and moreover that $\sigma(z)$ has no zeroes or poles on $\Sigma$. In this way we manage to produce explicit expressions for multi-loop string vacuum amplitudes entirely within the topological membrane approach.

\subsection{Examples: One-Loop Amplitudes}

While we have obtained expressions for the partition functions on any Riemann surface $\Sigma$, it is clear from the previous subsection that in general one needs to incorporate the gravitational sector of the topological membrane in order to properly deal with the effective wavefunctions. In this respect it is illuminating to examine them in some detail in the simplest case of genus $g=1$, where everything can be worked out explicitly and there is no gravitational anomaly to contend with. The resulting formulas can be deduced from the general equations that we have obtained in the previous subsection. This will serve, among other things, to illustrate explicitly how the three-dimensional formalism naturally reproduces modular invariants of the conformal field theories.

\section{- $\underline{\text { Torus }}$}

Let us begin with the torus $T^{2}$. In this case the period matrix may be represented by the Teichmüller modular parameter $\Gamma=\tau=\tau_{1}+i \tau_{2}$, a complex number with imaginary part $\tau_{2}>0$. The complex coordinates on $T^{2}$ are given by the identifications $z \equiv z+s+r \tau$, for any $s, r \in \mathbb{Z}$, in the complex plane, and the holomorphic harmonic one-form may be taken to be $\omega(z)=1$. The area of the torus is $\mathcal{A}_{\tau}=\tau_{2}$, and the determinant of the scalar Laplace operator on $T^{2}$ is given by

$$
\operatorname{det}^{\prime} \nabla_{\tau}^{2}=\tau_{2}|\eta(\tau)|^{4},
$$


where

$$
\eta(\tau)=\mathrm{e}^{\pi i \tau / 12} \prod_{r=1}^{\infty}\left(1-\mathrm{e}^{2 \pi i r \tau}\right)
$$

is the Dedekind function.

The partition function is therefore given by

$$
\begin{aligned}
Z_{T^{2}}(\tau) & =\sum_{\lambda=0}^{p q-1} \overline{\chi_{\bar{\lambda}}(\tau)} \chi_{\lambda}(\tau) \\
& =\frac{1}{\sqrt{\tau_{2}}|\eta(\tau)|^{2}} \sum_{\lambda=0}^{p q-1} \sum_{s \in p \mathbb{Z}} \sum_{s^{\prime} \in p \mathbb{Z}} \exp \left\{\frac{2 \pi i \tau}{k}\left(s+\frac{\lambda}{q}\right)^{2}\right\} \exp \left\{-\frac{2 \pi i \bar{\tau}}{k}\left(s+\frac{\bar{\lambda}}{q}\right)^{2}\right\}
\end{aligned}
$$

where the $\chi$ 's may be recognized as the simple characters of the relevant chiral $U(1)$ current algebras extended by chiral vertex operators. They are related to the gauge theory wavefunctions by

$$
\begin{aligned}
\chi_{\lambda}(\tau) & =k^{g / 4} \Psi_{\lambda}(\tau) \\
& =\frac{1}{\tau_{2}^{1 / 4} \eta(\tau)} \sum_{s \in p \mathbb{Z}} \exp \left\{\frac{2 \pi i \tau}{k}\left(s+\frac{\lambda}{q}\right)^{2}\right\} .
\end{aligned}
$$

For the various orbifold theories based on the torus as the double cover, we simply need to specialize the general equations obtained in the previous subsection.

\section{- $\underline{\text { Klein Bottle }}$}

The Klein bottle $K^{2}$ can be obtained from the torus with modular parameter $\tau=2 i \tau_{2}$ under the orbifold generated by the parity operator $\Omega^{\prime}$ introduced at the beginning of section 3. This operation preserves the diagonal $U(1)$ current of the conformal field theory which is the generator of translations along the target space circle. There are two types of orbifolds that one can form. For the $\Omega^{\prime} C T$ quotient we obtain an untwisted theory with partition function

$$
Z_{K^{2}}\left(\tau_{2}\right)=\frac{1}{2\left(2 \tau_{2}\right)^{1 / 4}\left|\eta\left(2 i \tau_{2}\right)\right|} \sum_{m=0}^{p q / 2-1} \sum_{s=-\infty}^{\infty} \exp \left\{-\frac{4 \pi \tau_{2}}{k}(p s+m)^{2}\right\},
$$

while for $\Omega^{\prime} T$ we obtain a twisted theory with partition function

$$
Z_{\tilde{K}^{2}}\left(\tau_{2}\right)=\frac{\sqrt{k}\left(2 \tau_{2}\right)^{1 / 4}}{2\left|\eta\left(2 i \tau_{2}\right)\right|} \sum_{n=0}^{p q / 2-1} \sum_{s=-\infty}^{\infty} \exp \left\{-\frac{4 \pi \tau_{2}}{k}\left(p s+\frac{k}{4} n\right)^{2}\right\} .
$$

The partition functions (6.32) and (6.33) are related by a modular transformation of the covering torus $T^{2}$. To see this, we replace the modulus $\tau$ in (6.33) by the new modulus

$$
\tilde{\tau}=-\frac{1}{2 \tau},
$$


and use the Poisson resummation formula

$$
\sum_{r=-\infty}^{\infty} \mathrm{e}^{-\pi g r^{2}-2 \pi i b r}=\frac{1}{\sqrt{g}} \sum_{s=-\infty}^{\infty} \mathrm{e}^{-\pi(s-b)^{2} / g} .
$$

Applying (6.35) twice (in $n$ and $s$ ) to the partition function (6.33), and using the modular transformation property

$$
\eta\left(-\frac{1}{\tau}\right)=\sqrt{-i \tau} \eta(\tau)
$$

of the Dedekind function $(\underline{6.29})$, we arrive at the relationship

$$
Z_{\tilde{K}^{2}}(\tau)=Z_{K^{2}}(\tilde{\tau})
$$

The surface $\tilde{K}^{2}$ exhibits the Klein bottle $K^{2}$ as a tube terminating at two cross-caps.

- $\underline{\text { Annulus }}$

We now start considering open orbifold surfaces. The annulus $C^{2}$ is generated by the orbifold of the torus $T^{2}$ with modular parameter $\tau=i \tau_{2}$ under the usual worldsheet parity operator $\Omega$. Again, there are two types of orbifolds. For the $\Omega C T$ quotient we obtain a theory with Neumann boundary conditions and partition function

$$
Z_{C^{2}}\left(\tau_{2}\right)=\frac{1}{2 \tau_{2}^{1 / 4}\left|\eta\left(i \tau_{2}\right)\right|} \sum_{m=0}^{p q / 2-1} \sum_{s=-\infty}^{\infty} \exp \left\{-\frac{2 \pi \tau_{2}}{k}(p s+m)^{2}\right\}
$$

while for $\Omega T$ we get a theory with Dirichlet boundary conditions and partition function

$$
Z_{\tilde{C}^{2}}\left(\tau_{2}\right)=\frac{\sqrt{k} \tau_{2}^{1 / 4}}{2\left|\eta\left(i \tau_{2}\right)\right|} \sum_{n=0}^{p q / 2-1} \sum_{s=-\infty}^{\infty} \exp \left\{-\frac{2 \pi \tau_{2}}{k}\left(p s+\frac{k}{4} n\right)^{2}\right\}
$$

Once again the two partition functions are related by a modular transformation. Replacing the modulus dependence in (6.39) with

$$
\tilde{\tau}=-\frac{2}{\tau}
$$

and using the modular transform (6.36), after two Poisson resummations (in $n$ and $s$ ) we arrive at the relation

$$
Z_{\tilde{C}^{2}}(\tau)=Z_{C^{2}}(\tilde{\tau})
$$

The surface $\tilde{C}^{2}$ is a cylinder with two boundaries. 
- Möbius Strip

Finally, the Möbius strip $M^{2}$ is obtained from the torus with $\tau=1 / 2+i \tau_{2}$ under the orbifold by the parity operation $\Omega$. For the $\Omega C T$ orbifold one has Neumann boundary conditions and partition function

$$
Z_{M^{2}}\left(\tau_{2}\right)=\frac{1}{2 \tau_{2}^{1 / 4}\left|\eta\left(\frac{1}{2}+i \tau_{2}\right)\right|} \sum_{m=0}^{p q / 2-1} \sum_{s=-\infty}^{\infty} \exp \left\{\frac{\pi i}{k}\left(1+2 i \tau_{2}\right)(p s+m)^{2}\right\},
$$

while for $\Omega T$ we obtain Dirichlet boundary conditions and partition function

$$
Z_{\tilde{M}^{2}}\left(\tau_{2}\right)=\frac{\sqrt{k} \tau_{2}^{1 / 4}}{2\left|\eta\left(\frac{1}{2}+i \tau_{2}\right)\right|} \sum_{n=0}^{p q / 2-1} \sum_{s=-\infty}^{\infty} \exp \left\{\frac{\pi i}{k}\left(1+2 i \tau_{2}\right)\left(p s+\frac{k}{4} n\right)^{2}\right\} .
$$

In this case the modular transformation (6.34) yields the relation

$$
Z_{\tilde{M}^{2}}(\tau)=Z_{M^{2}}(\tilde{\tau}) .
$$

The surface $\tilde{M}^{2}$ is the usual one-loop double twist diagram of open string theory.

\subsection{Remarks on Modular Invariance}

In this subsection we briefly comment on the structure of modular invariant partition functions constructed from the results obtained above. A more precise examination of modular invariant quantities will be presented elsewhere [59]. The basic point we wish to make here is that while the torus partition function (6.30) is a modular invariant of $T^{2}$, the modular transformations above of the orbifold partition functions map the direct-channel picture, corresponding to $\Omega C T$ type orbifolds with partition functions (6.32), (6.38) and (6.42), to the transverse-channel picture corresponding to $\Omega T$ type orbifolds with partition functions given by (6.33), (6.39) and (6.43). To build a modular invariant partition function $Z_{\text {inv }}$, it is necessary to sum over both twisted and untwisted sectors or both sectors corresponding to Neumann and Dirichlet boundary conditions to get [16]

$$
Z_{\mathrm{inv}}=Z_{\Sigma^{\Omega C}}+Z_{\Sigma^{\Omega}}
$$

Note that here, in order to avoid half-integer state multiplicities, we must add the statistical sums rather than averaging them. In the case of open strings, the partition function (6.45) should of course be understood only as a formal mathematical object which is invariant under modular transformations, because the theories corresponding to Neumann and Dirichlet boundary conditions live in different Hilbert spaces. Put differently, the direct channel amplitudes have character expansions with integer-valued coefficients which count the number of allowed open and unoriented string states, and thereby have natural interpretations as 
partition functions. On the other hand, in the transverse channel they correspond to amplitudes of a closed string reflecting from the two boundaries or crosscaps at the ends of the cylinder and in general contain non-integer character expansion coefficients. Thus the transverse channel amplitudes do not count states and have no interpretation as partition functions. It is not natural to require modular invariance of these amplitudes, as they have very different physical interpretations.

Nevertheless, the function (6.45) could have a more profound physical significance if one could find some sort of tunneling mechanism which interpolates between the two types of boundary conditions. This would have to be a mechanism which allowed a flow between superselection sectors of the Hilbert space. A physical system which possesses the same sort of characteristics is a proton-antiproton system. While the proton and antiproton correspond to different superselection sectors, a composite wavefunction corresponding to a proton-antiproton bound state may be formed. Of course the present open string case is somewhat different in nature, because here we don't have the analog of charge conjugation as we tunnel between the different sectors. We are not aware at present of any such process in open string theory.

The partition function (6.45) corresponds to a modification of the orthogonal projection in (3.7) to an operator which picks out modular invariant states. The appropriate operator acting on states of the three-dimensional gauge theory is given by

$$
\mathcal{P}_{\text {inv }}=\frac{1}{2}(\mathbb{1}+\Omega C T)+\frac{1}{2}(\mathbb{1}+\Omega T) .
$$

Note that (6.46) is not a projector. It can only be completed into an orthogonal projection by adding to (6.45) a contribution corresponding to the twisted torus states. In general, one cannot build a modular invariant partition function using projection operators, but rather only one which is invariant under the relative modular group of the Riemann surface [66]. This latter group is, however, trivial at one-loop order. The operator 6.46) also ensures that the partition function is explicitly invariant under T-duality, which at the level of the worldsheet theory exchanges winding and Kaluza-Klein modes, and therefore necessarily interchanges both pictures and partition functions. Furthermore, in addition to the prefactors computed above, in the case of open string worldsheets there should appear an extra overall multiplicative factor $N^{2}$, where $N$ is the number of Chan-Paton factors in the corresponding string theoretic photon Wilson line [67]. This issue is under current development [59].

\section{Acknowledgments}

We thank M. Bianchi and A. Sagnotti for helpful comments on the manuscript. P.C.F. is grateful for the hospitality of the Department of Mathematics of Heriot-Watt University, 
where this work was completed. P.C.F. and I.I.K. thank A. Kovner for helpful discussions. The work of P.C.F. is supported by Grant SFRH/BPD/5638/2001 from FCT (Portugal). The work of I.I.K. is supported by PPARC Grant PPA/G/0/1998/00567 and EU Grant FMRXCT960090. The work of R.J.S. is supported by a PPARC Advanced Fellowship. 


\section{References}

[1] A. Sagnotti, Open Strings and their Symmetry Groups, in: "Nonperturbative Quantum Field Theory", eds. G. 't Hooft, A. Jaffe, G. Mac, P.K. Mitter and R. Stora (Plenum Press, New York, 1988), pp. 521-528.

[2] A. Sagnotti, Closed Strings and their Open String Descendants, Phys. Rep. 184 (1989) $167-175$.

[3] M. Bianchi and A. Sagnotti, On the Systematics of Open String Theories, Phys. Lett. B247 (1990) 517-524.

[4] A. Sagnotti, Some Properties of Open String Theories, in: "Supersymmetry and Unification of Fundamental Interactions (SUSY 95)", eds. I. Antoniadis and H. Videau (Ed. Frontières, 1996), pp. 473-484, hep-th/9509080.

[5] A. Sagnotti, Surprises in Open String Perturbation Theory, Nucl. Phys. (Proc. Suppl.) 56B (1997) 332-343, hep-th/9702093.

[6] C.P. Burgess and T.R. Morris, Open Superstrings à la Polyakov, Nucl. Phys. B291 (1987) 256-284; Open and Unoriented Strings à la Polyakov, Nucl. Phys. B291 (1987) $285-333$.

[7] J.P. Rodrigues, One Loop Amplitudes for the Bosonic Open String: A First Quantized Approach, Phys. Lett. B178 (1986) 350-356; Nonorientable One Loop Amplitudes for the Bosonic Open String: Electrostatics on a Möbius Strip, J. Math. Phys. 28 (1987) 2669.

[8] J.A. Harvey and J.A. Minahan, Open Strings in Orbifolds, Phys. Lett. B188 (1987) $44-50$.

[9] G. Pradisi and A. Sagnotti, Open String Orbifolds, Phys. Lett. B216 (1989) 59-67.

[10] P. Hořava, Strings on World Sheet Orbifolds, Nucl. Phys. B327 (1989) 461-484.

[11] J. Govaerts, Quantum Consistency Of Open String Theories, Phys. Lett. B220 (1989) 77.

[12] N. Marcus and A. Sagnotti, Tree Level Constraints on Gauge Groups for Type I Superstrings, Phys. Lett. B119 (1982) 97.

[13] N. Marcus and A. Sagnotti, Group Theory from 'Quarks' at the Ends of Strings, Phys. Lett. B188 (1987) 58. 
[14] A. Sagnotti, Anomaly cancellations and Open String Theories, in: "From Superstrings to Supergravity", Erice 1992 Proceedings, pp. 116-125, hep-th/9302099.

[15] M. Bianchi and A. Sagnotti, The Partition Function of the SO(8192) Bosonic String, Phys. Lett. B211 (1988) 407-416.

[16] J. Polchinski, String Theory (Cambridge University Press, 1998).

[17] I.I. Kogan, The Off-Shell Closed String as Topological Open Membranes: Dynamical Transmutation of World Sheet Dimension, Phys. Lett. B231 (1989) 377.

[18] S. Carlip and I.I. Kogan, Quantum Geometrodynamics of the Open Topological Membrane and String Moduli Space, Phys. Rev. Lett. 64 (1990) 1487; Three-Dimensional Gravity and String Ghosts, Phys. Rev. Lett. 67 (1991) 3647-3649, hep-th/9110005.

[19] S. Carlip and I.I. Kogan, Three-Dimensional Topological Field Theories and Strings, Mod. Phys. Lett. A6 (1991) 171-181.

[20] I.I. Kogan, Quantum Mechanics on the Moduli Space from the Quantum Geometrodynamics of the Open Topological Membrane, Phys. Lett. B256 (1991) 369; Quantum Liouville Theory from Topologically Massive Gravity: $1+1$ Cosmological Constant as Square of $2+1$ Graviton Mass, Nucl. Phys. B375 (1992) 362.

[21] S. Carlip, Inducing Liouville Theory from Topologically Massive Gravity, Nucl. Phys. B362 (1991) 111-124.

[22] S. Carlip, (2+1)-Dimensional Chern-Simons Gravity as a Dirac Square Root, Phys. Rev. D45 (1992) 3584-3590, hep-th/9109006.

[23] L. Cooper and I.I. Kogan, Boundary Conditions and Heterotic Construction in Topological Membrane Theory, Phys. Lett. B383 (1996) 271-280, hep-th/9602062.

[24] G. Amelino-Camelia, I.I. Kogan and R.J. Szabo, Conformal Dimensions from Topologically Massive Quantum Field Theory, Nucl. Phys. B480 (1996) 413-456, hep-th/9607037; Gravitational Dressing of Aharonov-Bohm Amplitudes, Int. J. Mod. Phys. A12 (1997) 1043-1052, hep-th/9610057.

[25] L. Cooper, I.I. Kogan and K.-M. Lee, String Winding Modes from Charge NonConservation in Compact Chern-Simons Theory, Phys. Lett. B394 (1997) 67-74, hep-th/9611107.

[26] I.I. Kogan, Three-Dimensional Description of the $\Phi_{1,3}$ Deformation of Minimal Models, Phys. Lett. B390 (1997) 189-196, hep-th/9608031. 
[27] L. Cooper, I.I. Kogan and R.J. Szabo, Mirror Maps in Chern-Simons Gauge Theory, Ann. Phys. 268 (1998) 61-104, hep-th/9710179.

[28] L. Cooper, I.I. Kogan and R.J. Szabo, Dynamical Description of Spectral Flow in $N=2$ Superconformal Field Theories, Nucl. Phys. B498 (1997) 492-510, hep-th/9702088.

[29] I.I. Kogan and R.J. Szabo, Liouville Dressed Weights and Renormalization of Spin in Topologically Massive Gravity, Nucl. Phys. B502 (1997) 383-418, hep-th/9703071.

[30] I.I. Kogan and A. Lewis, Vacuum Instability in Chern-Simons Theory, Null Vectors and Two-Dimensional Logarithmic Operators, Phys. Lett. B431 (1998) 77-84, hep-th/9802102.

[31] I.I. Kogan, A. Momen and R.J. Szabo, Induced Dilaton in Topologically Massive Quantum Field Theory, J. High Energy Phys. 9812 (1998) 013, hep-th/9811006.

[32] P. Castelo Ferreira, I.I. Kogan and B. Tekin, Toroidal Compactification in String Theory from Chern-Simons Theory, Nucl. Phys. B589 (2000) 167-195, hep-th/0004078.

[33] P. Castelo Ferreira and I.I. Kogan, Open and Unoriented Strings from Topological Membrane: I. Prolegomena, J. High Energy Phys. 0106 (2001) 056, hep-th/0012188.

[34] P. Castelo Ferreira, Heterotic, Open and Unoriented Strings from Topological Membrane, D.Phil. Thesis - Oxford University (unpublished), hep-th/0110067.

[35] For a review see: I.I. Kogan, Lectures on Topological Membranes, in: "Particles and Fields", eds. J.C.A. Barata, A.P.C. Malbouisson and S.F. Novaes (World Scientific, Singapore, 1998), pp. 223-290; also available at: http://www-thphys.physics.ox.ac.uk/users/IanKogan/membrane.ps.

[36] J.F. Schonfeld, A Mass Term for Three-Dimensional Gauge Fields, Nucl. Phys. B185 (1981) 157.

[37] S. Deser, R. Jackiw and S. Templeton, Three-Dimensional Massive Gauge Theories, Phys. Rev. Lett. 48 (1982) 975-978; Topologically Massive Gauge Theories, Ann. Phys. 140 (1982) 372-411.

[38] G. Moore and N. Seiberg, Taming the Conformal Zoo, Phys. Lett. B220 (1989) 422.

[39] S. Elitzur, G. Moore, A. Schwimmer and N. Seiberg, Remarks on the Canonical Quantization of the Chern-Simons-Witten Theory, Nucl. Phys. B326 (1989) 108.

[40] E. Witten, Quantum Field Theory and the Jones Polynomial, Commun. Math. Phys. 121 (1989) 351-399. 
[41] M. Bos and V.P. Nair, U(1) Chern-Simons Theory and $c=1$ Conformal Blocks, Phys. Lett. B223 (1989) 61; Coherent State Quantization of Chern-Simons Theory, Int. J. Mod. Phys. A5 (1990) 959.

[42] J.M.F. Labastida and A.V. Ramallo, Operator Formalism for Chern-Simons Theories, Phys. Lett. B227 (1989) 92; Chern-Simons Theory and Conformal Blocks, Phys. Lett. B228 (1989) 214.

[43] W. Ogura, Path Integral Quantization of Chern-Simons Gauge Theory, Phys. Lett B229 (1989) 61.

[44] E. Witten, On Holomorphic Factorization of WZW and Coset Models, Commun. Math. Phys. 144 (1992) 189-212.

[45] P. Hořava, Chern-Simons Gauge Theory on Orbifolds: Open Strings from Three Dimensions, J. Geom. Phys. 21 (1996) 1-33, hep-th/9404101.

[46] L. Birke, J. Fuchs and C. Schweigert, Symmetry Breaking Boundary Conditions and WZW Orbifolds, Adv. Theor. Math. Phys. 3 (1999) 671-726, hep-th/9905038.

[47] G. Felder, J. Fröhlich, J. Fuchs and C. Schweigert, The Geometry of WZW branes, J. Geom. Phys. 34 (2000) 162-190, hep-th/9909030.

[48] L.R. Huiszoon, K. Schalm and A.N. Schellekens, Boundaries, Crosscaps and Simple Currents, hep-th/0007174.

[49] J. Fuchs and C. Schweigert, D-Brane Conformal Field Theory and Bundles of Conformal Blocks, math.qa/0004034.

[50] G. Felder, J. Fröhlich, J. Fuchs and C. Schweigert, Conformal Boundary Conditions and Three-Dimensional Topological Field Theory, Phys. Rev. Lett. 84 (2000) 16591662, hep-th/9909140.

[51] G. Felder, J. Fröhlich, J. Fuchs and C. Schweigert, Correlation Functions and Boundary Conditions in RCFT and Three-Dimensional Topology, hep-th/9912239.

[52] J. Fuchs and C. Schweigert, Solitonic Sectors, Conformal Boundary Conditions and Three-Dimensional Topological Field Theory, hep-th/0009111.

[53] J. Fuchs, I. Runkel and C. Schweigert, Conformal Correlation Functions, Frobenius Algebras and Triangulations, hep-th/0110133.

[54] J. Fuchs, I. Runkel and C. Schweigert, Conformal Boundary Conditions and 3D Topological Field Theory, hep-th/0110158. 
[55] L.R. Huiszoon, K. Schalm and A.N. Schellekens, Geometry of WZW Orientifolds, hep-th/0110267.

[56] I. Brunner, On Orientifolds of WZW Models and their Relation to Geometry, hep-th/0110219.

[57] C.P. Bachas, N. Couchoud and P. Windey, Orientifolds of the Three-Sphere, hep-th/0111002.

[58] M. Asorey, F. Falceto and S. Carlip, Chern-Simons States and Topologically Massive Gauge Theories, Phys. Lett. B312 (1993) 477-485, hep-th/9304081.

[59] P. Castelo Ferreira, I.I. Kogan and R.J. Szabo, in preparation.

[60] E. Witten, Topology Changing Amplitudes in (2+1)-Dimensional Gravity, Nucl. Phys. B323 (1989) 113-140.

[61] E.C. Marino, Quantum Theory of Nonlocal Vortex Fields, Phys. Rev. D38 (1988) 3194.

[62] A. Kovner, B. Rosenstein and D. Eliezer, Photon as a Goldstone Boson in $(2+1)$ Dimensional Abelian Gauge Theories, Nucl. Phys. B350 (1991) 325.

[63] A. Kovner and B. Rosenstein, Topological Interpretation of Electric Charge, Duality and Confinement in (2+1)-Dimensions, Int. J. Mod. Phys. A7 (1992) 7419.

[64] I.I. Kogan and A. Kovner, Compact QED in Three Dimensions: A Simple Example of a Variational Calculation in a Gauge Theory, Phys. Rev. D51 (1995) 1948.

[65] E. D'Hoker and D.H. Phong, The Geometry of String Perturbation Theory, Rev. Mod. Phys. 60 (1988) 917.

[66] M. Bianchi and A. Sagnotti, Open Strings and the Relative Modular Group, Phys. Lett. B231 (1989) 389.

[67] M. Bianchi, G. Pradisi and A. Sagnotti, Toroidal Compactification and Symmetry Breaking in Open String Theories, Nucl. Phys. B376 (1992) 365-386. 